\title{
Somite morphogenesis is required for axial blood vessel formation
}

Eric Paulissen, Nicholas J. Palmisano, Joshua Waxman, \& Benjamin L. Martin*

\section{Department of Biochemistry and Cell Biology}

Stony Brook University Stony Brook, NY 11794-5215

\section{*Correspondence: benjamin.martin@stonybrook.edu}

\section{Summary statement}

Retinoic acid induced somite morphogenesis generates a midline cavity that accommodates migrating angioblasts, which form the axial blood vessels.

\begin{abstract}
Angioblasts that form the major axial blood vessels of the dorsal aorta and cardinal vein migrate towards the embryonic midline from distant lateral positions. Little is known about what controls the precise timing of angioblast migration and their final destination at the midline. Using zebrafish, we found that midline angioblast migration requires neighboring tissue rearrangements generated by somite morphogenesis. The somitic shape changes cause the adjacent notochord to separate from the underlying endoderm, creating a ventral midline cavity that provides a physical space for the angioblasts to migrate into. The anterior to posterior progression of midline angioblast migration is facilitated by retinoic acid induced anterior to posterior somite maturation and the subsequent progressive opening of the ventral midline cavity. Our work demonstrates a critical role for somite morphogenesis in organizing surrounding tissues to facilitate notochord positioning and angioblast migration, which is ultimately responsible for creating a functional cardiovascular system.
\end{abstract}

Keywords: Retinoic acid, dorsal aorta, cardinal vein, somite, morphogenesis, ventral midline cavity, notochord endoderm separation, vasculogenesis, angioblasts, mesoderm, zebrafish 


\section{INTRODUCTION}

54 Early organismal development relies on a variety of tissues that collectively organize into

55 a functional body plan. In the trunk of the vertebrate embryo, many tissues are

56 differentiating at once, and often interact with one another to influence their definitive

57 structure (McMillen and Holley, 2015). The axial (notochord), paraxial (somites), and

58 lateral (angioblasts and other tissues) mesoderm begins to be specified during

59 gastrulation, and new cells are added to these tissues from posteriorly localized

60 progenitor cells as they expand after gastrulation (Kimelman, 2016; Martin, 2016; Martin

61 and Kimelman, 2012; Row et al., 2016). By the end of embryogenesis, the notochord is

62 a prominent midline tissue of the trunk, and provides structural support to the body and

63 signals that pattern adjacent tissues (Balmer et al., 2016; Glickman et al., 2003; Kimmel

64 et al., 1995). Pairs of somites flank either side of the notochord and give rise to tissues

65 that include the skeletal muscle, tendons, and bone (Tani et al., 2020). Ventral to the

66 notochord and between the somites, the axial vasculature structures of the dorsal aorta

67 and posterior cardinal vein distribute blood throughout the embryo (Hogan and Bautch,

68 2004; Isogai et al., 2001).

70 During somitogenesis, newly formed somites undergo a maturation event in which they

71 change their morphology from a cuboidal shape to a chevron shape, and extend in the

72 dorsal-ventral axis (Kimmel et al., 1995; Tlili et al., 2019). Many cellular rearrangements

73 and mechanical stresses contribute to making the final definitive somite shape (Hollway

74 et al., 2007; Leal et al., 2014; Tlili et al., 2019; Yin and Solnica-Krezel, 2007; Yin et al.,

75 2018; Youn and Malacinski, 1981). At the same time that somites are changing shape,

76 endothelial progenitors called angioblasts arise in the lateral plate mesoderm and

77 migrate to the midline of the embryo to form the axial vasculature (Jin et al., 2005).

78 Interestingly, angioblast migration and new somite formation occur in an anterior to

79 posterior progression, with a wave of angioblast migration happening slightly after the

80 wave of somitogenesis (Jin et al., 2005; Kohli et al., 2013; Yabe and Takada, 2016).

81 Despite these two events occurring in close temporal and physical proximity to one

82 another, it is not clear how they influence one another during this developmental stage.

83 While some evidence has shown somite-related defects on blood vessel patterning,

84 much of this research focused on angiogenic sprouting or defects in arterial-venous

85 specification, well after angioblast migration and somitogenesis is completed (Lawson et 
86 al., 2002; Shaw et al., 2006; Therapontos and Vargesson, 2010; Torres-Vázquez et al.,

87 2004).

88

89 Loss of function mutation of the notochord specifying gene noto gene results in non-

90 autonomous migration defects of angioblasts as they move to the midline. (Fouquet et

91 al., 1997; Helker et al., 2015). In the absence of noto, the notochord adopts a somitic

92 fate (Fouquet et al., 1997; Talbot et al., 1995). The requirement of notochord

93 specification for angioblast migration suggested that the notochord may act to attract

94 angioblasts to the midline. This model was further explored when a notochord-derived

95 secreted factor named apela (also known as toddler/elabela) was discovered (Chng et

96 al., 2013; Freyer et al., 2017; Helker et al., 2015; Pauli et al., 2014). This small peptide is

97 expressed in the notochord and when mutated caused angioblast migration defects.

98 However, apela loss of function also causes defects in mesoderm and endoderm

99 formation prior to notochord and angioblast specification, via gastrulation defects (Freyer

100 et al., 2017; Norris et al., 2017; Pauli et al., 2014). Similarly, noto loss of function causes

101 morphological changes that could interfere with angioblast migration, including a broad

102 expansion of somite tissue near the developing blood vessels (Halpern et al., 1995).

103 Thus, although it appears that mutations that affect notochord development can disrupt

104 angioblast migration, the exact mechanism of this effect is not clear.

105

106 As new somites form during body axis extension, they secrete all-trans retinoic acid

107 (RA). RA is a metabolic derivative of vitamin A, and a series of alcohol and aldehyde

108 dehydrogenases convert Vitamin A into RA in specific locations of the embryo including

109 newly formed somites (Duester, 2008). RA acts as a morphogen with broad roles during

110 vertebrate embryogenesis. Some of these include the development of the limb, skeleton,

111 hindbrain, and heart (Emoto et al., 2005; Heine et al., 1986; Lohnes et al., 1994;

112 Mendelsohn et al., 1994; Niederreither et al., 2001; Sandell et al., 2007). Although

113 involved in many processes, RA has a particular influence over the maturation of

114 mesoderm and somitic tissue (Hamade et al., 2006; Janesick et al., 2018, 2014; Li et al.,

115 2015). While RA is notably involved in ensuring the bilateral symmetry of the somites, it

116 is ultimately dispensable for axis elongation in the zebrafish (Berenguer et al., 2018;

117 Bernheim and Meilhac, 2020; Hamade et al., 2006; Kumar and Duester, 2014). Based

118 on the timing of somite derived RA signaling activity relative to when angioblasts migrate

119 to the midline, we speculated it may be involved in midline angioblast migration. 
121 Here we show that RA mediated somite maturation is required for the proper formation

122 of the axial vasculature through a non-autonomous role in inducing morphological

123 changes in surrounding tissues. A process we call notochord-endoderm separation

124 (NES) occurs prior to angioblast migration, wherein the dorsal translocation of the

125 notochord during development leads to its separation from the endoderm along the

126 dorsal-ventral axis to generate a transient cavity we refer to as the ventral midline cavity

127 (VMC). The angioblasts migrate toward and eventually into the VMC after NES. The

128 induction of NES and the VMC is somite maturation dependent, and a delay or failure in

129 NES can cause systemic angioblast migration defects. This evidence places somite-

130 maturation as a critical event that is required for NES and the development of the axial

131 vasculature.

\section{RESULTS}

\section{Retinoic acid promotes convergence of angioblasts to the midline and the}

\section{6 formation of the axial vasculature}

138 To determine if RA signaling impacts angioblast migration, we made time-lapse movies

139 of $t g(k d r l: G F P)$ embryos, which express GFP in angioblasts (Jin et al., 2005). Embryos

140 were mounted at the 10 somite-stage and their migration was observed over a 3-hour

141 time period. Wild-type angioblasts migrated to the midline in a manner consistent with

142 previously described research (Figure 1A, Supplemental Movie 1) (Helker et al., 2015;

143 Jin et al., 2005; Kohli et al., 2013). To determine what effect the loss of RA would have

144 on the migrating angioblasts, we generated aldh1a2 mutants with the $\operatorname{tg}(k d r l: G F P)$

145 angioblast reporter in the background. Time-lapse imaging of these embryos shows

146 defects in angioblast migration to the midline (Figure 1B, Supplemental Movie 2), which

147 was also confirmed by in-situ hybridization (Figure S1). Three other methods of

148 disrupting RA signaling caused the same phenotype. Loss of RA by treatment with N,N-

149 diethylaminobenzaldehyde (DEAB) (an inhibitor of the Aldh family, including Aldh1a2

150 (Morgan et al., 2015)), the small molecule retinoic acid receptor (RAR) alpha and

151 gamma inhibitor (BMS453), or expression of a dominant negative RARa using the

$152 T g(h s p 701: E G F P-d n H s a . R A R A)$ transgenic line (hereafter referred to as HS:dnRAR) all

153 caused midline migration defects (Skvarca et al., 2019) (Figure S1). Angioblasts in these 
154 embryos show delayed migration with disorganized anterior to posterior processivity. To

155 determine if addition of RA accelerated angioblast migration as well as anterior to

156 posterior processivity, we compared the angioblast migration patterns of 10 somite stage

$157 \operatorname{tg}(k d r l: G F P)$ embryos treated with either DMSO (vehicle) or $0.1 \mu \mathrm{M}$ RA at the tailbud

158 stage (Figure 1C,1D, respectively). Angioblasts in the DMSO treated embryos migrate

159 normally, but the angioblasts treated with RA had prematurely initiated migration and

160 arrived earlier to the midline during development (Figure 1C and Figure 1D, respectively,

161 Supplemental Movie 3). To determine the rate at which angioblasts reach the midline,

162 we quantified the percentage of GFP fluorescence at the midline in relation to the total

163 amount of fluorescence for the both wild-type and aldh1a2 -/. We defined angioblast

164 fluorescence as reaching the midline if they are settled beneath the notochord. The

165 aldh1a2 -/- embryos show very low percentage fluorescence at the midline, relative to

166 total fluorescence, compared to wild-type embryos 4 hours after observation, when

167 angioblast migration is largely complete (Figure 1E). On the other hand, fluorescence

168 was higher at the midline in embryos treated with RA than in DMSO treated control

169 embryos (Figure 1F). This shows that RA is necessary and sufficient for promoting

170 angioblast migration to the midline. To determine at which stage RA is needed for

171 angioblasts to migrate to the midline, we administered DEAB at different stages of

172 development. Inhibition of RA at tailbud stage caused the angioblast migration defect,

173 but treatment at the 5-somite stage resulted in normal migration (Figure $1 \mathrm{H}$ and $1 \mathrm{l}$,

174 respectively), thus establishing the critical developmental window for RA signaling

175 regulation of midline angioblast migration. 

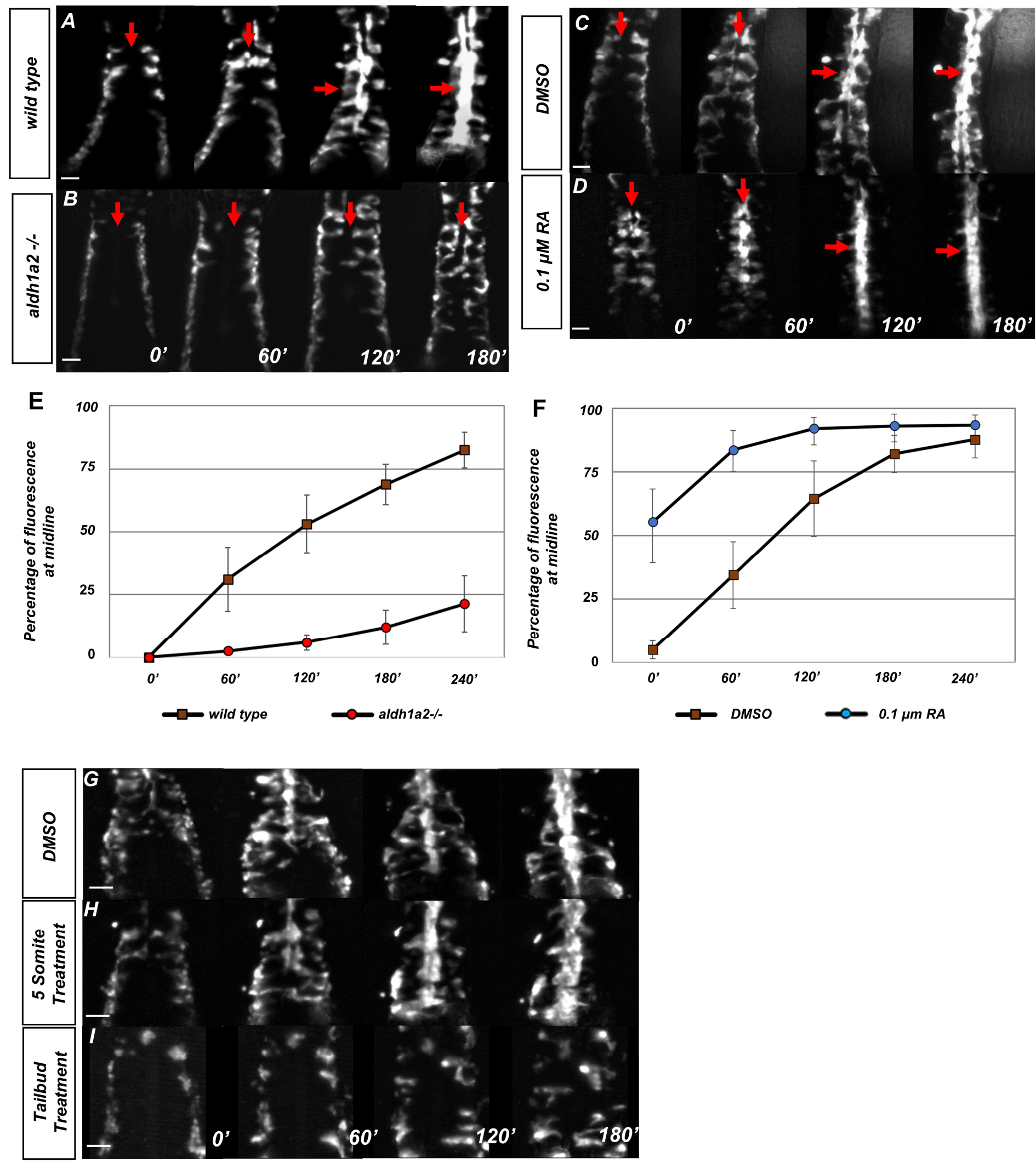

176 
Figure 1.Retinoic acid is required prior to segmentation for angioblast migration.

(A-D) Dorsal view of $\operatorname{tg}(k d r l: e G F P)$ embryos with representative images taken every 60 minutes over the course of 180 minutes, in $(A)$ untreated $(N=12$, see Supplemental Movie 1), (B) aldh1a2 -/- mutants ( $\mathrm{N}=12$, see Supplemental Movie 2), (C) DMSO treated $(\mathrm{N}=10)$, or $0.1 \mu \mathrm{M}$ RA treated embryos $(\mathrm{N}=8$, see Supplemental Movie 3$)$. The anterior of the embryo is at the top and red arrows indicate the midline. (E, F) Graph of fluorescence intensity as angioblasts arrive at the midline for (E) wild-type and aldh1a2 $-/$ - and for (F) DMSO and $0.1 \mu \mathrm{M}$ RA. Y-axis indicates percentage of fluorescent intensity at midline relative to the fluorescence intensity of whole embryo. Time is measured on the $\mathrm{X}$-axis. $\mathrm{N}=8$ for all conditions, error bars indicate standard deviation. (G-I) time-lapse images of $t g(k d r l: e G F P)$ embryos treated with either (G) DMSO vehicle $(n=10)$ or $(\mathbf{H}) 20 \mu \mathrm{M}$ DEAB at the 5 somite stage $(n=6)$, and (I) $20 \mu \mathrm{M}$ DEAB at tailbud stage $(n=14)$. Scale bars, $50 \mu \mathrm{m}$.

178 Retinoic acid is required cell non-autonomously for angioblast migration.

180 To determine if RA functions cell autonomously to induce angioblast migration, we

181 utilized the transgenic zebrafish line $T g$ (hsp70l:id3-2A-NLS-KikGR), which carries a heat

182 shock inducible construct that overexpresses id3 after a temperature shift (Row et al.,

183 2018). We previously showed that overexpression of $i d 3$ from this line can cause

184 transplanted cells from a donor embryo to faithfully adopt an endothelial fate in a wild-

185 type host embryo. (Row et al., 2018). We utilized this by crossing Tg(hsp70l:id3-2A-NLS-

186 KikGR)), hereafter referred to HS:id3, to HS:dnRAR, to generate donor embryos

187 wherein transplanted cells would be targeted to the endothelium and express dominant

188 negative retinoic acid receptors (RARA). Cells positive for both transgenic constructs

189 were transplanted into $\operatorname{tg}(k d r l: e G F P)$ host embryos and these cells exhibited normal

190 migration to the midline (Figure 3A, Supplemental Movie 4). In addition, we utilized a RA

191 reporter line, $\operatorname{tg}(R D B D, 5 X U A S: G F P)$, to determine which tissues were subject to RA

192 signaling (Mandal et al., 2013). We performed immunohistochemistry against GFP and

193 the transcription factor Etv2, which labels the angioblasts (Figure 3B) (Sumanas et al.,

194 2005). The GFP expression indicates tissues that have been exposed to RA. These

195 tissues included the adaxial region of the somites, the notochord, and epidermis (Figure

196 3B, white arrow, white arrowhead, and gold arrow, respectively). The primitive

197 angioblasts, labeled with anti-Etv2 staining, do not overlap with GFP staining (Figure 3B,

198 red arrows). Together these results indicate that angioblasts are not receiving an RA

199 signal and do not require RA signaling for migration when surrounded by a wild-type

200 environment. 
bioRxiv preprint doi: https://doi.org/10.1101/2021.04.07.438831; this version posted October 21, 2021. The copyright holder for this preprint

(which was not certified by peer review) is the author/funder, who has granted bioRxiv a license to display the preprint in perpetuity. It is made available under aCC-BY-NC-ND 4.0 International license.

\section{1}

202 We investigated which tissue was responsible for the angioblast migration defect based

203 on the observed activity of the reporter. Given its proximity to the midline, we chose to
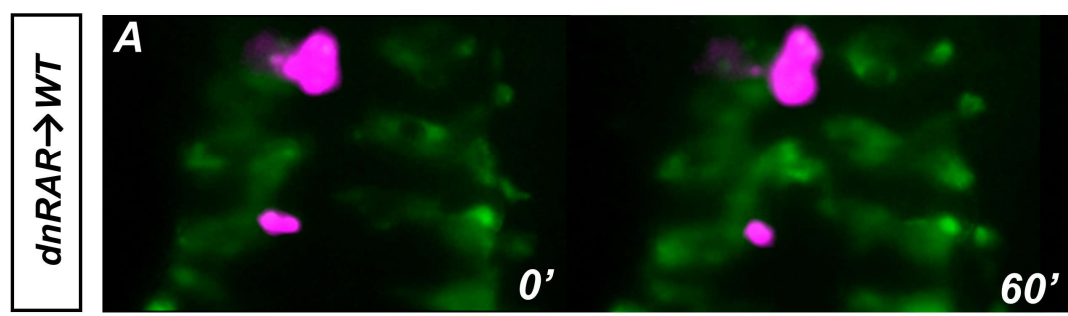

$120^{\prime}$

$180^{\prime}$
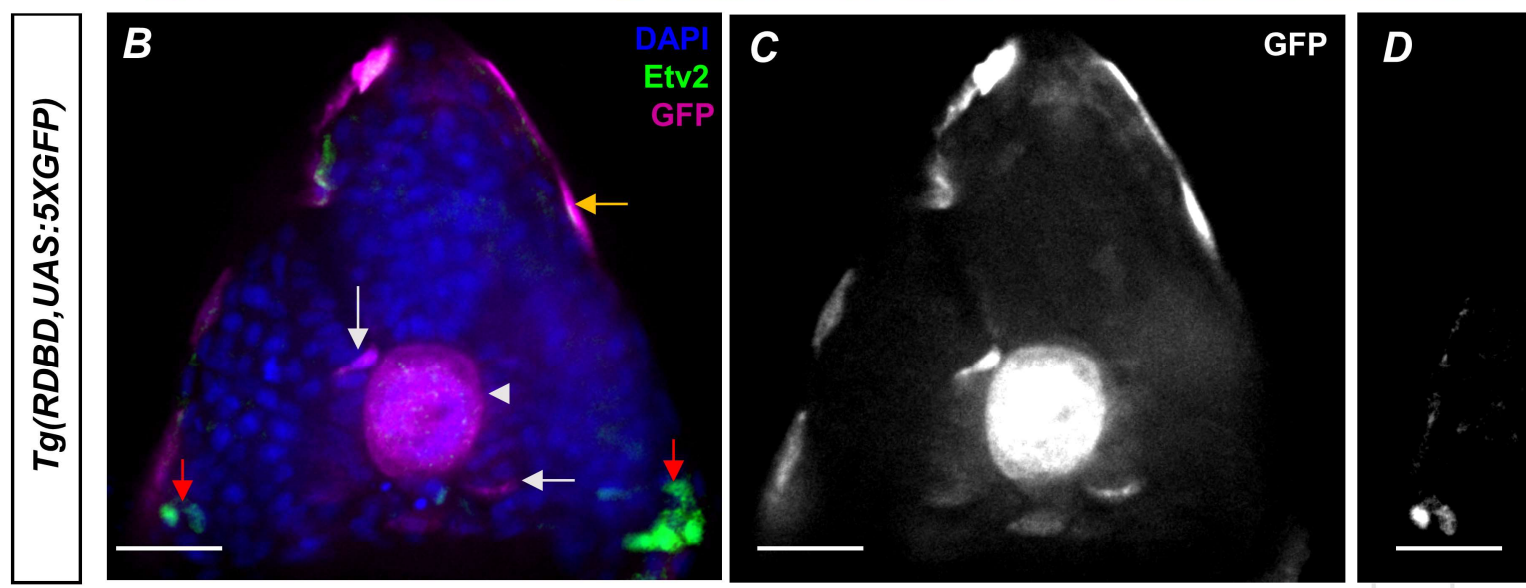

Etv2
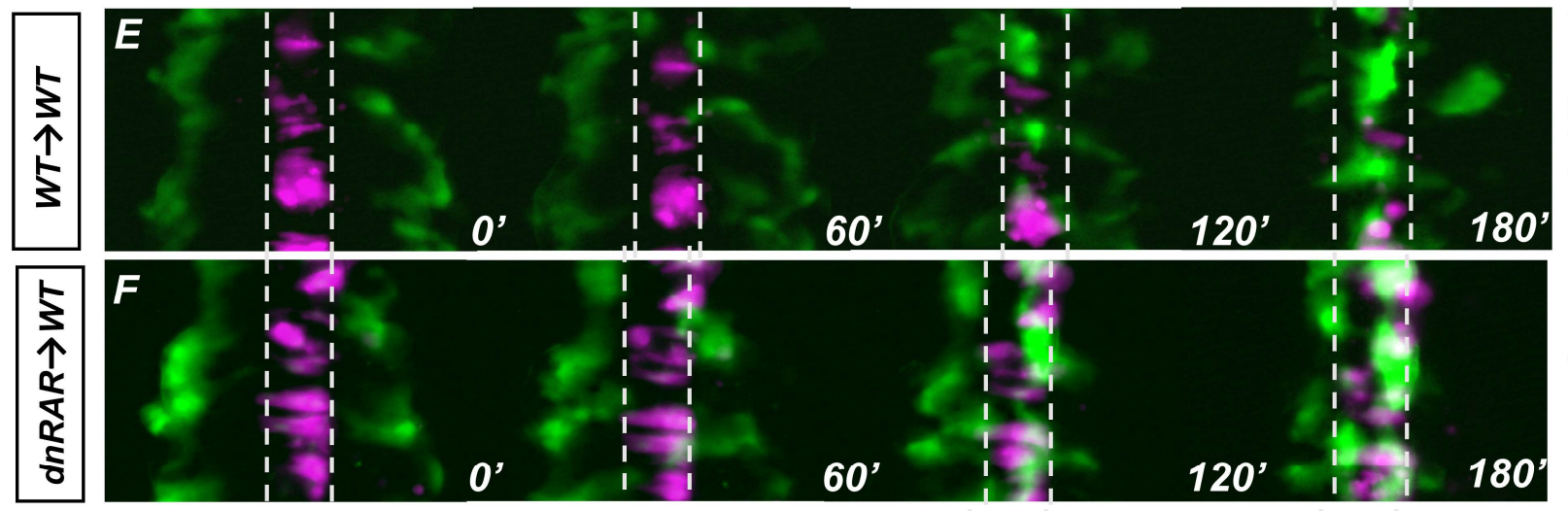

\begin{tabular}{|l}
\hline 5 \\
5 \\
5 \\
5
\end{tabular}
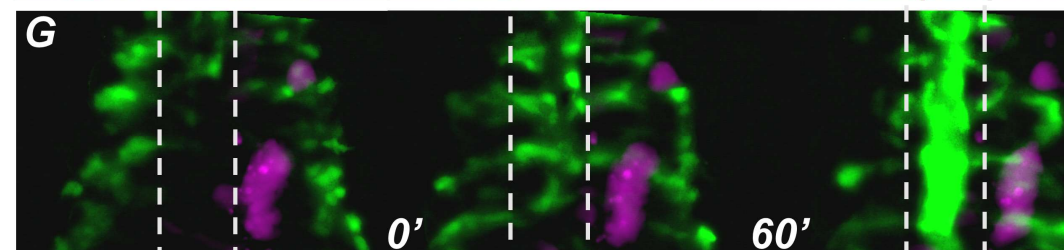

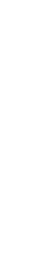

占
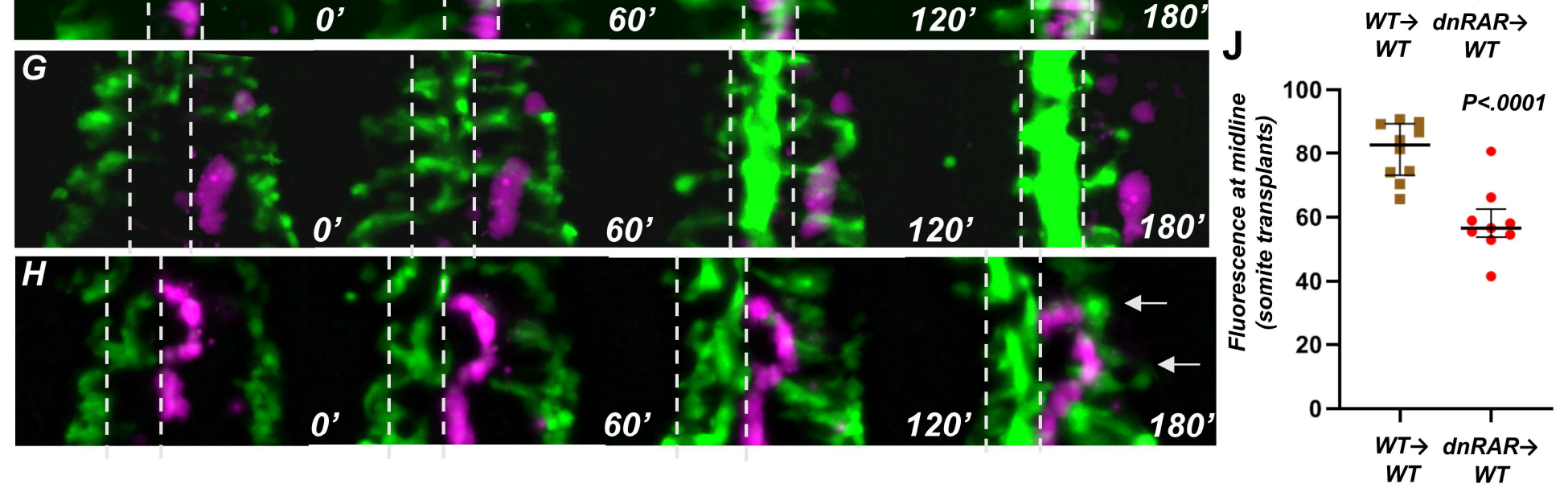


\section{Figure 2. Retinoic acid signaling is not present in the endothelium and is required in the somites for angioblast migration.}

(A) Representative images of a time-lapse of $\operatorname{tg}(H S$ : $d n R A R), \operatorname{tg}(H S: i d 3)$ cells transplanted into a wild-type embryo. Cells labeled in magenta indicate migrating cells. These cells migrate along with the host angioblasts labeled by $t g(k d r l: g f p)$, indicating normal migration patterns in absence of cell-autonomous RA depletion conditions ( $n=3$ ) (see Supplemental Movie 4). (B) DAPI stained section of 13somite-stage $\operatorname{tg}(R D B D, 5 X U A S: G F P)$ embryo, immunostained for GFP and Etv2 $(n=12)$. GFP signal is present in adaxial region of the somite, the notochord, and the epidermis (white arrow, white arrowhead, and gold arrows, respectively). The red arrows indicate areas of Etv2 staining. (C) GFP channel of image in (B). (D) Etv2 channel of image in (B). Note the lack of overlap between Etv2 staining and

GFP staining. (E) Representative images of a time-lapse of wild-type cells transplanted into a wild-type notochord. (F) Representative images of a time-lapse of $\operatorname{tg}(H S: d n R A R)$ cells transplanted into a wild-type notochord. (G) Representative images of a time-lapse of wild-type cells transplanted into a wild-type somite. $(\mathbf{H})$ Representative images of a time-lapse of $\operatorname{tg}(H S: d n R A R)$ cells transplanted into a

wild-type somite. White arrows indicate angioblast migration defects. (I) Quantification of midline fluorescence, as a percentage of total, for notochord transplants at 180'. The difference for wild-type and $\operatorname{tg}(H S: d n R A R)$ were not statistically significant $(\mathrm{P}=0.254)$. ( $\mathrm{J})$ Quantification of midline fluorescence, as a percentage of total, for somite transplants at 180'. The difference for wild-type and

$\operatorname{tg}(H S: d n R A R)$ were statistically significant $(\mathrm{P}<0.0001)$. Scale bars, $50 \mu \mathrm{m}$.

test whether the notochord or somitic mesoderm contributed to the defect. To test notochord contribution, we transplanted cells from a wild-type donor to a $\operatorname{tg}(\mathrm{kdrl}$ :eGFP)

206 host embryo to target the midline progenitors (Row et al., 2016). Host embryos with wild-

207 type cells transplanted to the notochord displayed normal angioblast migration to the

208 midline (Figure 2E). Similarly, transplants of $\operatorname{tg}(H S: d n R A R)$ cells into the notochord

209 showed normal midline angioblast migration(Figure 2F). In addition, in-situ hybridizations

210 against a known notochord-secreted angioblast chemoattractant apela, as well as the

211 receptors aplnra and aplnrb, show little change in RA depleted conditions (Figure

212 Supplement 2A-2F). This indicates that the lack of RA signaling in notochord cells is not

213 causing the angioblast migration defects. We then transplanted cells to target the somitic

214 mesoderm. Wild-type cells transplanted into the somitic mesoderm showed normal

215 angioblast migration to the midline (Figure $2 \mathrm{G}$ ). However, when we transplanted

$216 \operatorname{tg}(H S: d n R A R)$ cells into the somitic mesoderm, angioblasts showed migratory defects in

217 the region of the host embryo in which they were transplanted (Figure $2 \mathrm{H}$ ).

218 Quantification of fluorescence percentage at the midline, in the chimeric regions of the

219 notochord, showed no statistically significant difference compared to controls (Figure 2l). 
220 However, quantification of fluorescence percentage in the chimeric regions of the

221 somites showed statistical significance between wild-type and $\operatorname{tg}(H S: d n R A R)$ (Figure

222 2J). This indicates that the somites are the principal tissue required for RA signaling

223 mediated midline angioblast migration.

The somitic mesoderm is required for angioblast migration to the midline.

To follow up the somite and notochord targeted transplant results, we examined midline

228 angioblast migration in embryos where these tissues are absent. Previous studies have 229 investigated genes linking the notochord to angioblast migration. Mutants of the genes

230 noto and apela were found to be notochord specific genes required for proper angioblast 231 migration (Cleaver and Krieg, 1998; Fouquet et al., 1997; Helker et al., 2015). The noto

232 gene induces notochord, and the notochord in turn secretes Apela which attracts the

233 angioblasts to the midline. Noto is required for the formation of the notochord, and in the

234 absence of Noto, the midline cells adopt a somitic fate (Talbot et al., 1995).

235 However, it is not clear if midline convergence was delayed or absent in notochord-less

236 embryos. In noto mutants, some angioblasts reach the midline at later developmental

237 stages, and resolve into a single blood vessel (Fouquet et al., 1997). We injected a noto

238 morpholino that faithfully phenocopies the noto mutant into $t g(k d r l: G F P)$ embryos

239 (Ouyang et al., 2009). Control morphant embryos show normal angioblast migration

240 (Figure 3A), whereas noto morphants show slowed angioblast migration (Figure 3B).

241 However, midline convergence still occurs in both conditions (Figure 3C). This implies

242 that the notochord is ultimately dispensable for the formation of the VMC and midline

243 positioning of angioblasts.

245 Having ruled out the role of notochord tissue, we next investigated whether the somitic

246 mesoderm is required for NES and the formation of the VMC. Previous studies have

247 shown that the t-box transcription factor $t b \times 16$ is required for proper somite formation

248 (Amacher et al., 2002; Goto et al., 2017; Griffin et al., 1998; Kimmel et al., 1989;

249 Manning and Kimelman, 2015; Row et al., 2011). In the absence of Tbx16, cells that

250 would normally join the paraxial mesoderm fail to do so, causing a deficiency in the trunk

251 somitic mesoderm, but leaving the endothelium intact (Thompson et al., 1998). We

252 utilized $t b \times 16$ mutants to determine whether the lack of somites caused angioblast

253 migration defects, similar to RA loss of function. We examined midline angioblast 
254 migration in $t b x 16$ mutant embryos with the $t g(k d r l: G F P)$ transgene in the background. In

255 wild-type sibling embryos, angioblast migration progresses normally to the midline

256 (Figure 3D). However, in tbx16 -/- embryos, angioblast migration does not occur, and

257 they remain in their positions in the lateral plate (Figure 6E). Fluorescence quantification

258 indicated that angioblasts never reach the midline in any embryo (Figure 3F). To

259 determine the effect of either notochord or somite loss on the definitive vasculature, we

260 performed transverse sections of $\operatorname{tg}(\mathrm{kdrl}: G F P)$ embryos injected with control, noto, and

261 tbx16 morpholinos and stained with DAPI. Control morpholino showed normal formation

262 of the dorsal aorta and common cardinal vein (Figure 3G). Embryos injected with noto

263 morpholino have angioblasts located at the midline that appear to have resolved into one

264 blood vessel structure (Figure $3 \mathrm{H}$ ). Embryos injected with tbx16 morpholinos, however,

265 show two distinct vessels located on either side of the notochord (Figure 3I). This

266 indicates that the somites are critical for angioblast convergence at the midline, while the

267 notochord is ultimately dispensable.

268

269 

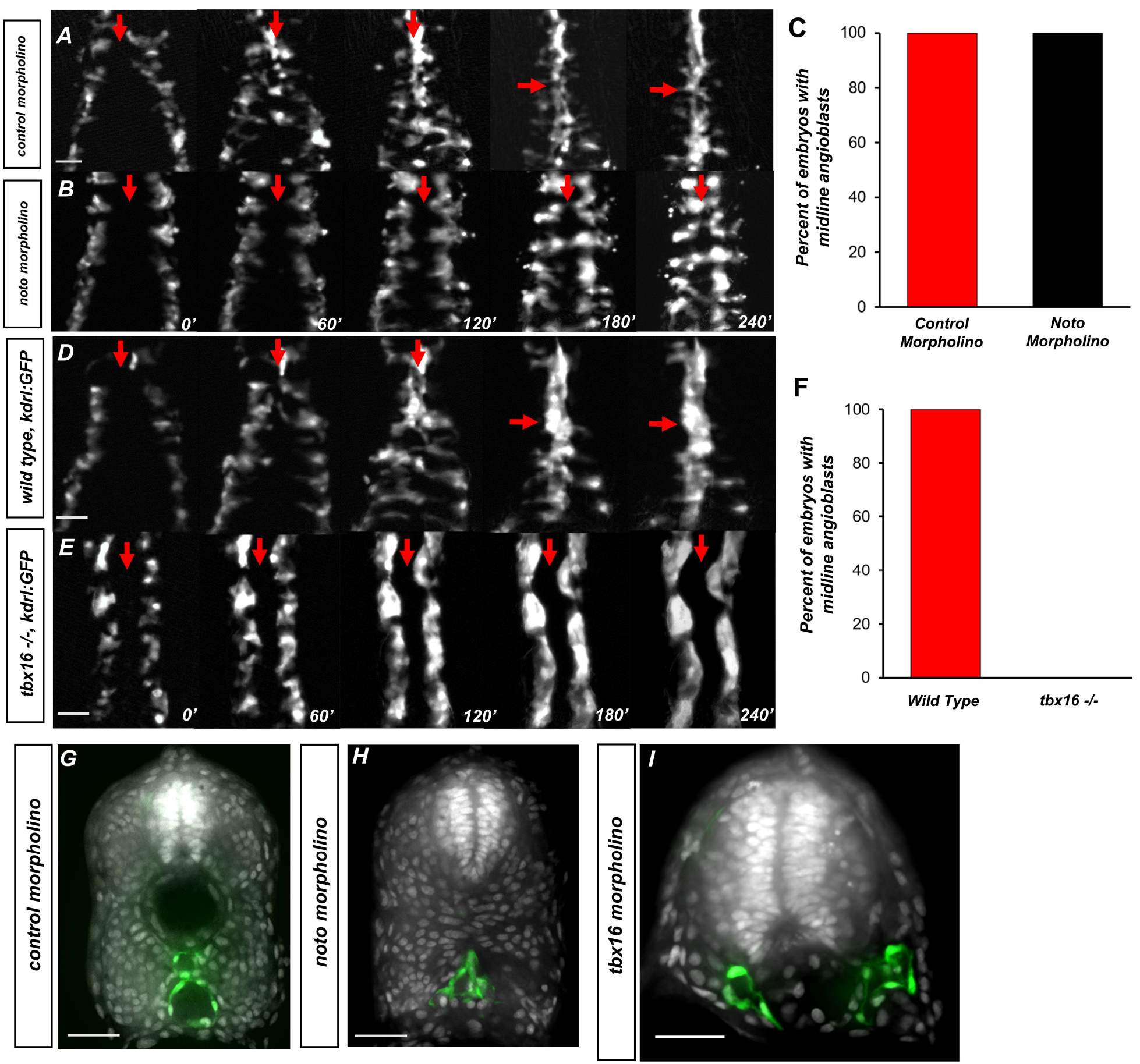

270 


\section{Figure 3. The somitic mesoderm, not the notochord, is required for midline convergence of angioblasts.}

(A) Time-lapse imaging of $t g(k d r l: e G F P)$ embryos injected with control morpholino over a 240' period. (B) tg(kdrl:eGFP) embryos injected with noto morpholino over a

240' period. Red arrows indicate the midline. (C) Graph of embryos with midline angioblasts in noto and control morphants after 240'. $\mathrm{N}=15$ for both conditions (D)

Time-lapse imaging of wild-type, tg(kdrl:eGFP) embryos over a 240' period. (E)

Timelapse imaging of $t b \times 16-/-, \operatorname{tg}(k d r l: e G F P)$ embryos over a 240' period. Red arrows indicate the midline. (F) Graph of embryos with midline angioblasts in wildtype and tbx16 -/- after 240' N =18 for both conditions. (G) Section of wild-type, tg(kdrl:eGFP) embryos over a 240' period. (H) Section of $24 \mathrm{hpf} t g(k d r l: e G F P)$ embryos injected noto morpholino and stained with DAPI. (I) Section of $24 \mathrm{hpf}$ $\operatorname{tg}(k d r l: e G F P)$ embryos injected $t b \times 16$ morpholino. Blood vessels are labeled in green and nuclei labeled in grey. Scale bars, $50 \mu \mathrm{m}$.

\section{Retinoic acid induces a dorsal translocation of the notochord away from the}

\section{3 underlying endoderm.}

275 Given the non-autonomous midline angioblast migration defects in RA loss of function

276 embryos, we examined if RA manipulation altered the normal development of non-

277 angioblast midline tissues. We utilized embryos labeled with a cell surface marker,

$278 \operatorname{tg}(u b b: I c k-m n g)$ (Adikes et al., 2020), and found the relative location of notochord at the

279 midline changes based on retinoic acid activity. To measure this, embryos were treated

280 with either DMSO, DEAB, or RA at the tailbud stage. The embryos were then fixed,

281 deyolked, and imaged in the trunk at roughly the $5^{\text {th }}$ somite (Figure $4 A-C$ ). The relative

282 location of the notochord in DMSO treated embryos was in close proximity to the ventral

283 underlying endoderm at the 12-somite stage, but that distance increased by the 15-

284 somite stage (Figure 4A). In DEAB treated embryos, the notochord remains ventrally

285 localized at both the 12-somite stage and 15-somite stage (Figure 4B). When the same

286 experiment was done with embryos exposed to exogenous RA, the notochord was

287 prematurely dorsally localized at the 12-somite stage and extended even further by the

288 15-somite stage (Figure 4C).

290 We then determined the dynamics of the notochord displacement in real time. Using DIC 291 images, we were able to overlay the lateral trunk with the notochord. We then observed

292 the notochord displacement in a 250' time-lapse for DMSO treated wild-type, aldh1a2 -/-

293 with DMSO, and exogenous RA (Figure 4D, 4E, and 4F, respectively). We quantified this

294 data by measuring the distance of the ventral aspect of the notochord to the yolk cells of 
295 the embryo (yellow brackets). We found that aldh1a2 -/- embryos showed delayed dorsal

296 displacement relative to DMSO in both the timing of dorsal movement and the

297 magnitude of that movement (Figure 4G).

299 Previous work showed that as somites mature, they extend in the both the dorsal and

300 ventral axis (Tlili et al., 2019), and based on this we hypothesized this extension was

301 concurrent with the dorsal translocation of the notochord. However, we wanted to rule

302 out the effect of forces on the anterior-most and posterior-most regions of the notochord.

303 The notochord is a rigid structure that is anchored in the tailbud near the notochord

304 primordia, and extends into the head mesoderm medial to the otic vesicle (Kimmel et al.,

305 1995). To eliminate the contribution other tissues could have on NES, we generated

306 trunk explants from embryos containing both $\operatorname{tg}$ (actc1b:gfp) and tg(tbxta:kaede)

307 transgenese that contained roughly 10 somites of the embryo. The 10-somite stage

308 embryos were sectioned near the somite borders of the $1^{\text {st }}$ and $10^{\text {th }}$ somite. We

309 performed live imaging of these trunk explants, focusing on the immature somites of the

310 posterior region (white boxes) (Figure 4H-4J, Supplemental Movie 5). In order to better

311 visualize the notochord, we separated the $\operatorname{tg}($ actc1b:gfp) and $\operatorname{tg}(t b x t a: k a e d e)$ signals into

312 separate channels. This distinguished the notochord reporter tg(tbxta:kaede) (Figure

$3134 \mathrm{H}^{\prime}-4 \mathrm{~J}$ '), from the somite reporter tg(actc1b:gfp) (Figure 4H"-4J"). The notochord reporter

314 showed clear dorsal movement away from the auto-fluorescent yolk (Figure 4H'-J'). The

315 somites also showed the corresponding expansion along the dorsal-ventral axis (Figure

$3164 \mathrm{H}^{\prime \prime}-4 \mathrm{~J}$ "). Together, this data indicates that the proper development of the midline is both

317 retinoic acid-dependent and requires only factors localized to the trunk of the embryo. 

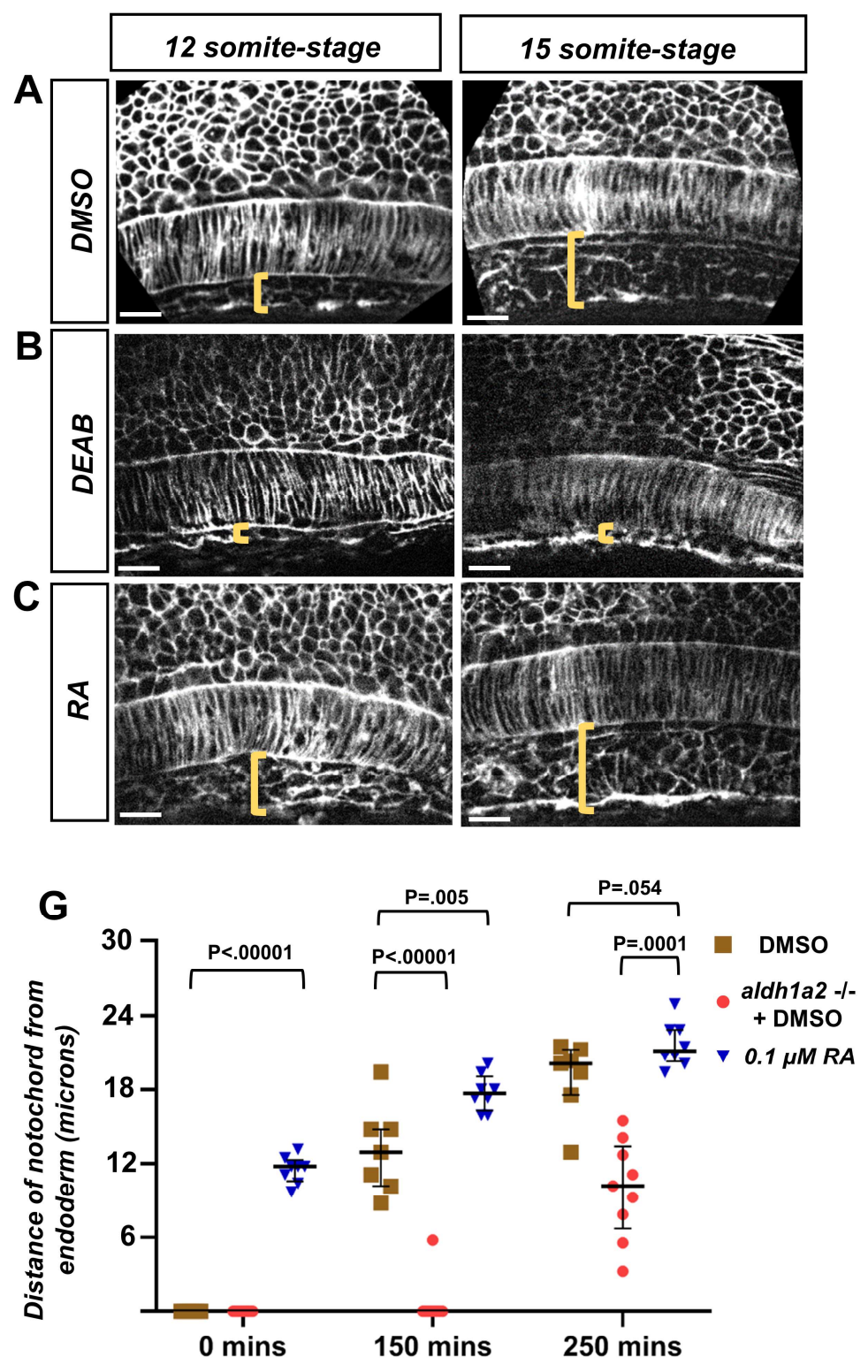

318

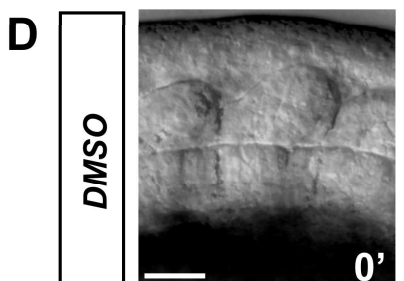

E
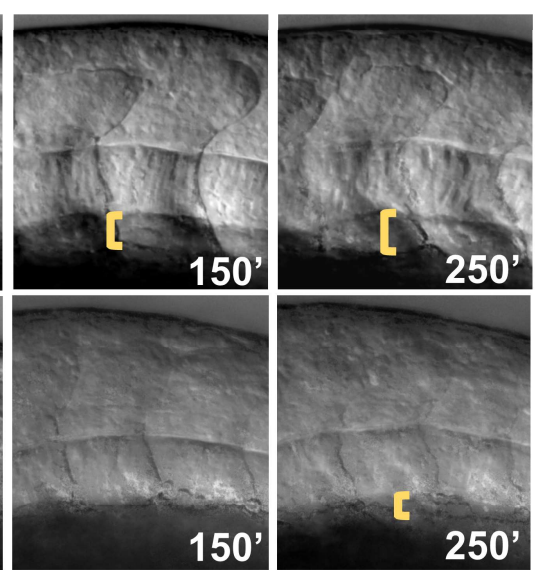

$\mathbf{F}$
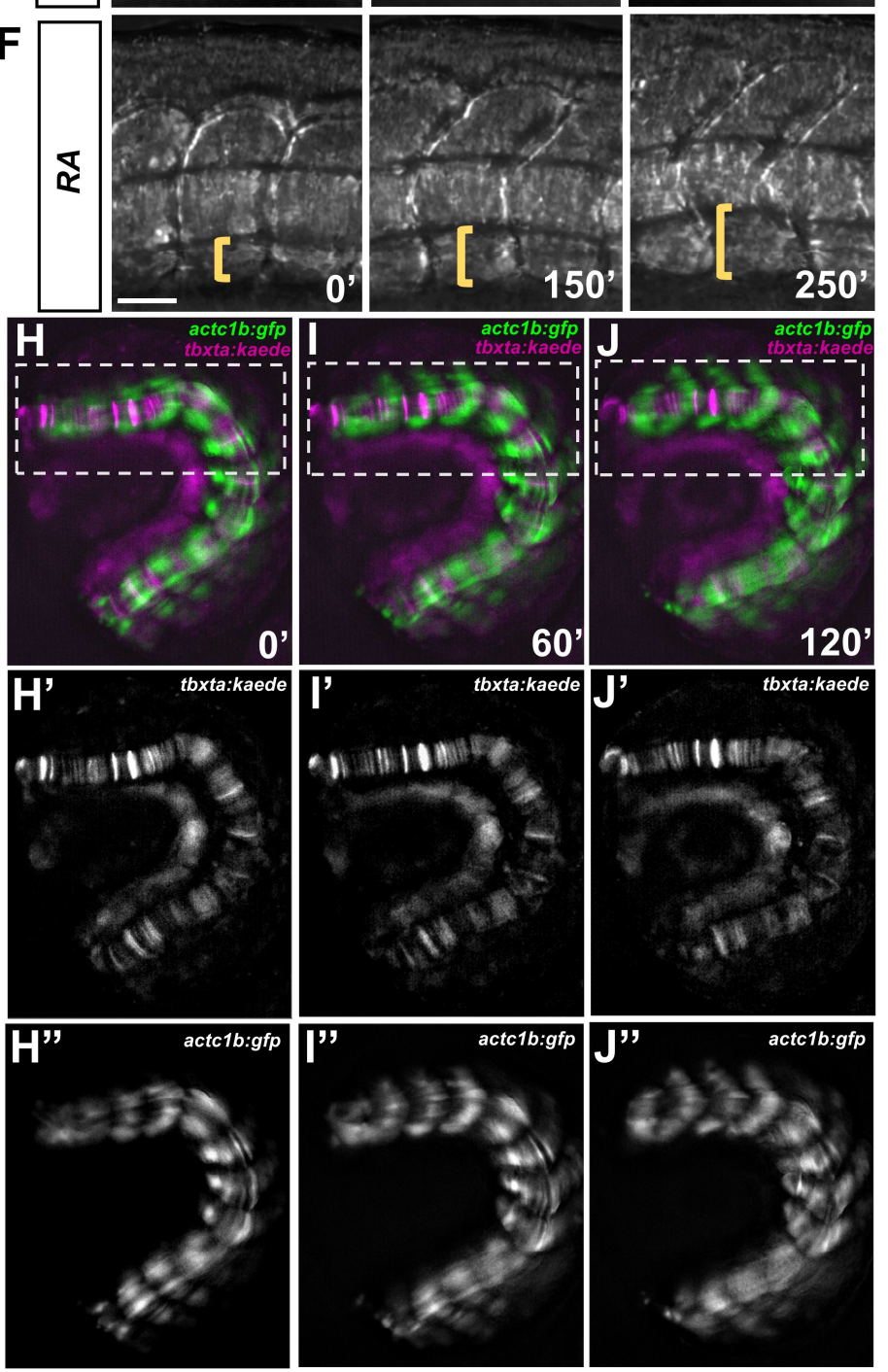


\section{Figure 4: RA signaling promotes dorsal translocation of the notochord}

(A-C) Fluorescent images of fixed (A) DMSO treated (B) DEAD treated and (C) RA treated tg(ubb:lck-mng) embryo trunks at the 12- and 15-somite stage. Yellow brackets indicate the distance between the notochord and underlying yolk cell. (D-F) DIC time-lapse images of the notochord and zebrafish trunk. Focal planes of the notochord and somites were overlaid to show the relative location to one another. Yellow brackets indicate the distance of the ventral notochord to the ventral-most portion of the embryo in (D) DMSO treated embryos, (E) aldh1a2 -/- embryos treated with DMSO, or (F) $0.1 \mu \mathrm{M} \mathrm{RA}$ treated embryos. (G) Quantification of dorsal translocation of notochord from the ventral tissue. Black lines indicate the median and interquartile range. Wildtype are indicated by brown squares, while aldh1a2 -/- and $0.1 \mu \mathrm{M} \mathrm{RA}$ embryos are shown by red circles and blue triangles, respectively. At $\mathrm{t}=0$,

DMSO vs. RA is $P<.00001$. At 150', DMSO is $P<.00001$ and $P=.005$ vs. aldh1a2 -/- and RA treatment, respectively. At 250', DMSO is $P=.0001$ and .054 vs. aldh1a2 -/- and RA treatment, respectively. (H-J) Time-lapse image of a trunk explant from tg(actc1b:gfp), tg(tbxta:kaede) embryo at (H) 0', (I) 60', and (J) 120'. (H'-J') Time-lapse image of explant showing tg(tbxta:kaede) only. (H'-J') Time-lapse image of explant showing tg(actc1b:gfp) only. Time-lapse shows the notochord adjacent the yolk at time 0', but moves dorsally from the yolk at time 120'. Scale bars, $25 \mu \mathrm{m}$.

320 Notochord-endoderm separation facilitates the midline convergence of

321 angioblasts

323 We speculated that RA dependent changes at the midline could be the cause of the

324 angioblast migration defect. In order to test this, we generated a heat-shock inducible

325 transgenic reporter of F-actin, $\operatorname{tg}(\mathrm{hsp} 70 \mathrm{l}$ :lifeact-mScarlet), which labels filamentous actin

326 throughout the embryo and allows visualization of all cells (Riedl et al., 2008). We

327 hereafter refer to $\operatorname{tg}$ (hsp70l:lifeact-mScarlet) as $\operatorname{tg}(H S$ :lifeact). We then crossed this

328 reporter to the $\operatorname{tg}(\mathrm{kdrl}: \mathrm{e} G F P)$ line to label the angioblasts. We treated embryos with

329 either DMSO (Figure 5A-5C), exogenous RA (Figure 5D and 5E), or DEAB (Figure 5F-

$3305 \mathrm{I}$ ). Embryos were sectioned at the region of the $5^{\text {th }}$ somite (Figure $5 \mathrm{~A}-5 \mathrm{I}$ ). Embryos that

331 were treated with DMSO showed normal midline angioblast migration patterns from the

332 12-somite stage, 15-somite stage, and 18-somite stage (Figure 5A-5C). As angioblasts

333 approach the midline, a gap appears between notochord/hypochord and the underlying

334 endoderm (Figure 5A). This gap is filled by angioblasts by the 15-somite stage,

335 separating the notochord from the endoderm (Figure $5 \mathrm{~B}$ ). We call the process of dorsal

336 translocation of the notochord the notochord-endoderm separation (NES). The transient 
337 opening that forms from NES is referred to as the ventral midline cavity (VMC). As

338 angioblast migration continues, additional angioblasts occupy the VMC until the 18-

339 somite stage, when angioblast migration is largely complete for the $5^{\text {th }}$ somite region of

340 the trunk (Figure 5C).

341

342 To determine if activation of retinoic acid is sufficient to accelerate angioblast migration

343 and NES concurrently, we treated embryos with exogenous RA and sectioned them at

344 the 12-somite and 15-somite stage. Embryos sectioned at the 12-somite stage showed

345 premature angioblast migration to the midline and NES, indicating that angioblast

346 migration and NES are responsive to exogenous RA signal (Figure 5D). Angioblasts

347 continue to be at the midline at 15-somite stage (Figure 5E). 18-somite stage embryos

348 were not available as posterior elongation discontinued in prolonged RA exposure, as

349 previously shown (Martin and Kimelman, 2010). To determine whether angioblast

350 migration and NES was attenuated in RA depletion conditions embryos were treated

351 with $D E A B$ and sectioned at the 12-somite, 15-somite, 18-somite, and 21-somite stage

352 (Figure 5F-5I respectively). At the 12-somite stage, the angioblasts are localized in the

353 lateral plate mesoderm (Figure 5F). Focusing on the midline of the embryo, we observe

354 that the notochord remains flush against the endoderm with no visible VMC or NES

355 (Figure 5G). At the 15-somite stage, we observe some midline movement of the

356 angioblasts, however the notochord remains flush against the endoderm and no

357 angioblasts have been able to reach the midline (Figure 5G). During the 18-somite

358 stage, a small VMC has formed between the notochord and endoderm. The leading

359 angioblasts have partially migrated into this VMC, however the bulk of the angioblasts

360 still reside outside of the midline (Figure $5 \mathrm{H}$ ). By the 21-somite stage, the angioblasts

361 have largely resolved to the midline, although with some angioblasts still residing outside

362 the midline (Figure 5I).

363 Given the pronounced delays in NES formation for anterior regions in RA depleted

364 embryos, we suspected that NES was a consequence of the known processive somite

365 maturation that begins in the anterior regions and progresses posteriorly (Stern and

366 Piatkowska, 2015). We sectioned 15-somite stage $\operatorname{tg}$ (HS:lifeact), $\operatorname{tg}($ kdrl:egfp) embryos

367 in the anterior and posterior regions corresponding to the $5^{\text {th }}$ and $12^{\text {th }}$ somite (Figure $5 \mathrm{~J}$ ).

368 In more immature $12^{\text {th }}$ somite, angioblasts reside in their lateral regions and NES is not

369 pronounced (white dashed lines outline the somites, and yellow brackets show the

370 distance from the notochord to the ventral endoderm) (Figure $5 \mathrm{~K}$ ). In the $5^{\text {th }}$ somite of 
371 the same embryo, angioblasts reside at the midline and NES is very pronounced,

372 indicating NES occurs anterior to posteriorly (Figure $5 \mathrm{~L}$ ). The lack of angioblasts at the

$37312^{\text {th }}$ somite, and low NES, is similar to DEAB treated embryos sectioned at the more

374 mature $5^{\text {th }}$ somite position (Figure $5 \mathrm{M}$ ).

375

376 To quantify how the dorsal translocation of the notochord was correlated with

377 angioblasts arriving to the midline, We created a two variable graph to measure both the

378 distance of the notochord to the ventral endoderm and the percentage of angioblasts

379 arriving at the midline for a given somite. For this analysis, we used 15-somite stage

380 embryos sectioned at the $5^{\text {th }}$ somite and $12^{\text {th }}$ somite. We expect that immature somites

381 in the posterior would have little midline migration of angioblasts and the anterior, with

382 more mature somites, would have significant midline migration. We measured the

383 distance in microns on the $x$-axis and the percentage of midline angioblasts on the $y$-axis

384 (Figure 50-5P). In DMSO treated embryos, embryos sectioned the $5^{\text {th }}$ somite grouped

385 together, showing both significant notochord-endoderm distance and angioblasts

386 residing at the midline (Figure 50, blue dots in the orange box). Embryos sectioned at

387 the $12^{\text {th }}$ somite show short notochord-endoderm distance and little angioblast migration

388 towards the midline (Figure 50, red squares in grey box). When comparing angioblasts

389 migration, the $5^{\text {th }}$ somite and $12^{\text {th }}$ somite regions were significantly different $(P>.0001)$.

390 The corresponding sectioning of DEAB treated embryos shows a difference compared

391 to wild-type in the $5^{\text {th }}$ somite region. Embryos sectioned at the $5^{\text {th }}$ somite have a small

392 distance from the notochord to endoderm, as well as no angioblast migration to the

393 midline (Figure 5P, blue dots in orange box). Interestingly, the notochord-endoderm

394 distance/migration percentage ratio strongly resembled the more immature somites. The

395 angioblast migration percentage was not statistically significant between the $5^{\text {th }}$ and $12^{\text {th }}$

396 somite in RA depletion conditions $(P=.33)$. This indicates that the midline exists in a

397 more immature state in RA depleted conditions, and the more mature state provides

398 space in which the angioblasts can migrate into. 

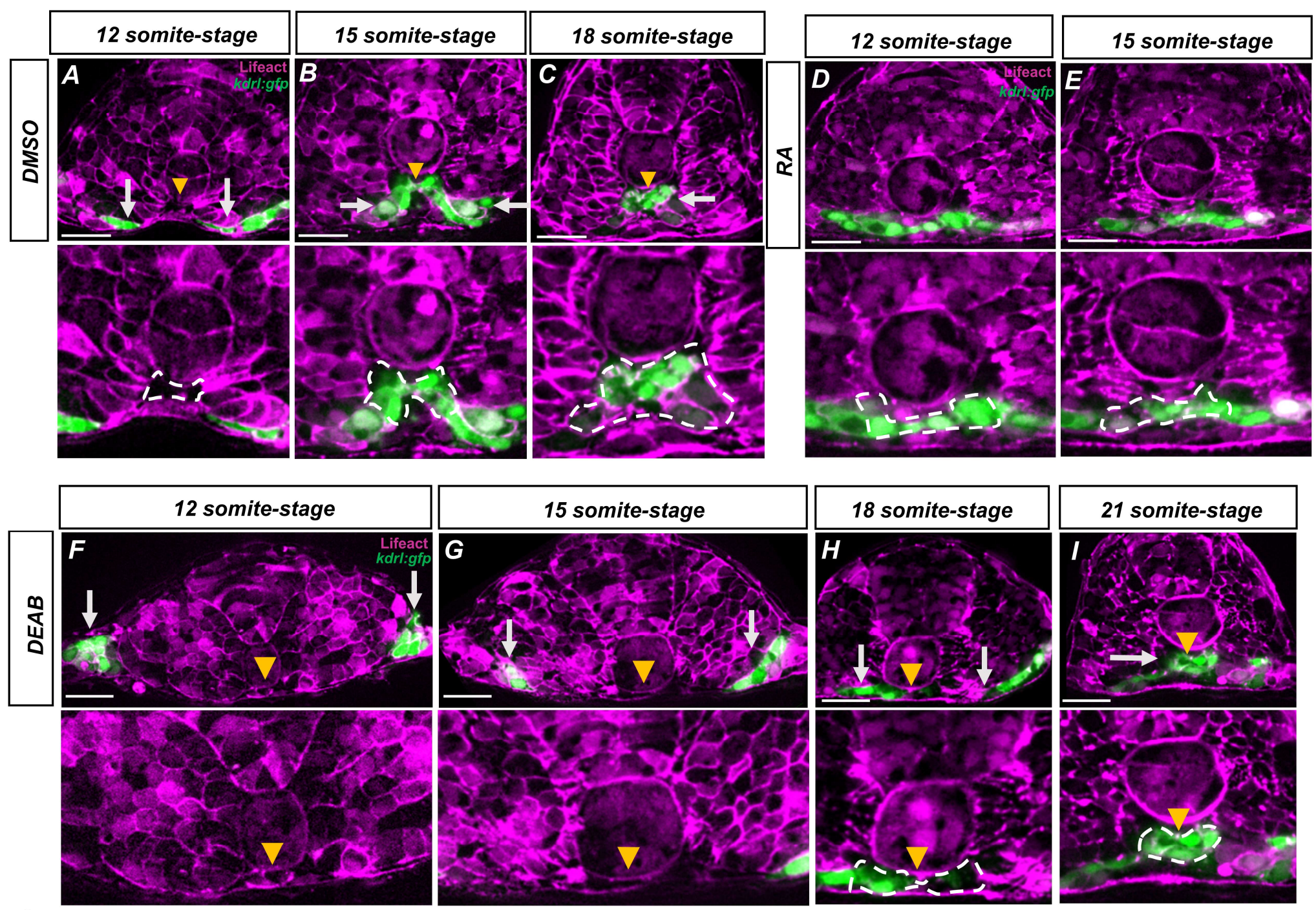

$\mathrm{J}$
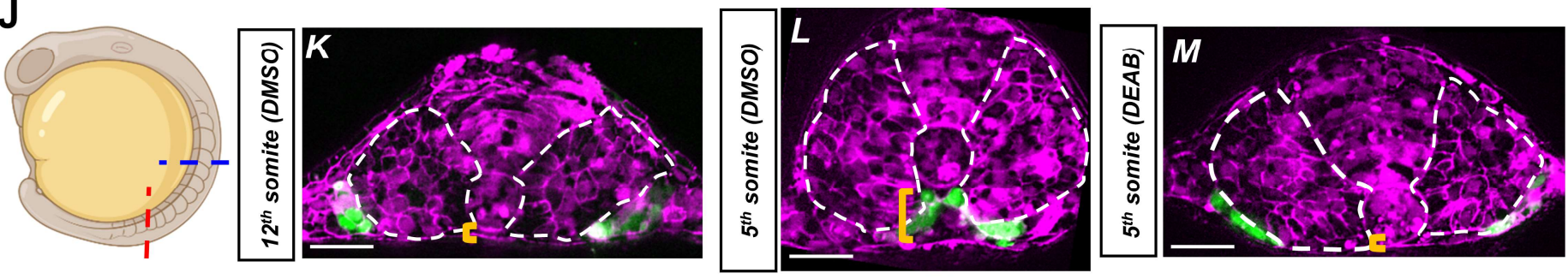

N

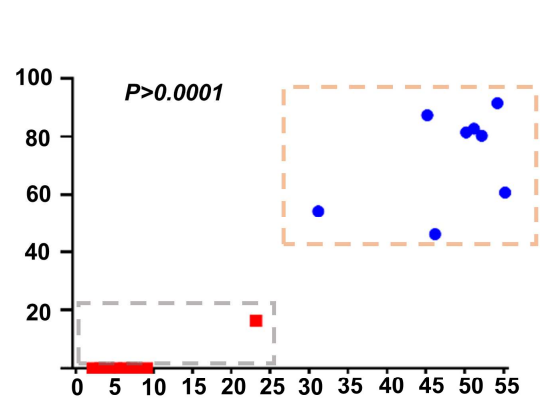

0

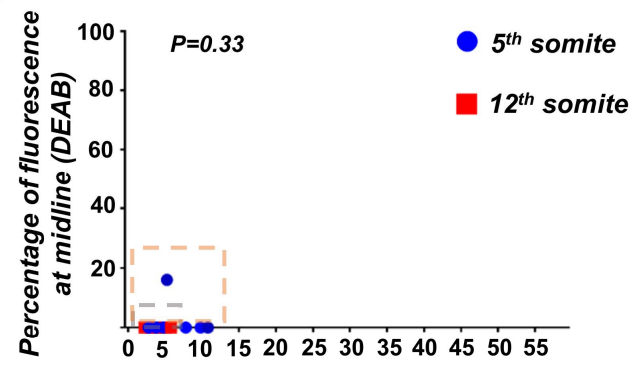

Distance of notochord to ventral endoderm $(\mu M)$

Distance of notochord to ventral endoderm $(\mu M)$ 


\section{Figure 5. Retinoic acid mediates a notochord-endoderm separation required for terminal angioblast migration.}

(A-I) Embryos generated by crossing $\operatorname{tg}(H S$ :lifeact) to $\operatorname{tg}(k d r l: e G F P)$ to label both actin and angioblasts. Yellow arrowheads indicate the midline while white arrows indicate angioblasts. A magnified image includes a white dashed line to indicate notochord-endoderm separation. (A-C)) DMSO treated embryos sectioned at the $5^{\text {th }}$ somite during the $($ A) 12 -somite stage $(n=8),(B) 15$ somite stage $(\mathrm{N}=8)$, and $(\mathrm{C}) 18$-somite stage $(\mathrm{N}=6)$. (D-E) $0.1 \mu \mathrm{M}$ RA treated embryos sectioned at the $5^{\text {th }}$ somite during the (D) 12-somite stage $(\mathrm{N}=8)$ and $(\mathrm{E}) 15$-somite stage $(\mathrm{n}=8)$. (F-I) $20 \mu \mathrm{M}$ DEAB treated embryos sectioned at the $5^{\text {th }}$ somite during the $(\mathbf{F}) 12$-somite stage $(N=7),(\mathbf{G})$ the 15 -somite stage $(\mathrm{N}=8)$, $(\mathbf{H}) 18$-somite stage $(\mathrm{N}=6)$, and $(\mathbf{I}) 21$-somite stage $(\mathrm{N}=6)$. ( $\mathbf{J}-\mathrm{L}) 15$ somitestage embryos generated by crossing $\operatorname{tg}(H S$ :lifeact) to $\operatorname{tg}(K d r l: e G F P)$. White dashed lines indicate outline of somites while yellow brackets indicate distance of notochord to ventral endoderm. (J) Schematic showing the sectioned region for experiments in (K-O). Red line indicates sectioning at $5^{\text {th }}$ somite and the blue line indicates sectioning at the $12^{\text {th }}$ somite. (K) A DMSO embryo sectioned at the $12^{\text {th }}$ somite. Note the lack of midline angioblasts and small distance between the notochord

and the ventral endoderm. (L) A DMSO-treated embryo sectioned at the $5^{\text {th }}$ somite. Note the angioblasts at the midline and larger distance from the notochord to the ventral endoderm. (M) A DEAB embryo sectioned at the $5^{\text {th }}$ somite. Note the lack of midline angioblasts and small distance between the notochord and the ventral endoderm. (N) A two variable graph showing percentage of angioblast fluorescence at the midline compared to distance between the notochord and the ventral endoderm. Blue dots indicate DMSO treated, 15-somite stage embryos sectioned at the $5^{\text {th }}$

somite and red squares are the same embryos sectioned at the $12^{\text {th }}$ somite $(N=8)$. ( 0$)$ A two variable graph showing percentage of angioblast fluorescence at the midline compared to distance between the notochord and the ventral endoderm. Blue dots indicate DEAB treated, 15-somite stage embryos sectioned at the $5^{\text {th }}$ somite and red squares are the same embryos sectioned at the $12^{\text {th }}$ somite $(\mathrm{N}=8)$. Scale bars, $50 \mu \mathrm{m}$.

402 Retinoic acid induces changes in the definitive vasculature.

404 Pharmacological inhibition of RA signaling has previously been shown to effect the

405 zebrafish vasculature, notably causing a smaller than normal dorsal aorta (Pillay et al.,

406 2016). To confirm this effect in the aldh1a2 -/- mutant, we sectioned $24 \mathrm{hpf} \operatorname{tg}(\mathrm{kdrl}: G F P)$

407 and aldh1a2 -/- embryos, stained the sections with DAPI, and imaged them using

408 spinning disk confocal microscopy (Figure 6A, 6B). Images show that the dorsal aorta

409 and posterior cardinal vein in wild-type embryos are roughly equivalent in size (Figure

$4106 \mathrm{~A}$, white arrows). However, in the aldh1a2 -/- embryos, the size of the dorsal aorta is

411 reduced but remained lumenized (Figure 6B, white arrows). We confirmed this

412 observation by in-situ hybridization of the arterial marker cldn5b and the venous marker

413 dab2, which showed similar effects (Figure S2A-D) (Casie Chetty et al., 2017). To

414 determine if this trend extended beyond the $24 \mathrm{hpf}$ developmental stage, we imaged the 
415 trunk of $5 \mathrm{dpf} t g(k d r l: G F P)$ embryos along with $\operatorname{tg}(k d r l: G F P)$, aldh1a2 -/- siblings (Figure

416 6C,6D). The $\operatorname{tg}(k d r l: G F P)$ embryos alone showed no vascular defects and normal dorsal

417 aorta and posterior cardinal vein (Figure 6C, white arrow and yellow arrowhead

418 respectively). However, the vasculature of the aldh1a2 -/- siblings show a large reduction

419 of the size of the dorsal aorta (Figure 6D, white arrow). In addition, it appears that the

420 posterior cardinal vein of aldh1a2 -/-increased in size compared to its sibling (Figure 6D,

421 yellow arrowhead). To determine if the size change was the result of a reduced cell

422 number in the DA in response to RA loss, we utilized the transgenic line $\operatorname{tg}(\mathrm{kdrl}: \mathrm{n} / \mathrm{s}$ -

423 GFP), which labels the nucleus of endothelial cells to count the number of cells in each

424 blood vessel over a $225 \mu \mathrm{M}$ horizontal section of a $24 \mathrm{hpf}$ zebrafish embryo. Embryos

425 were treated with either DMSO, or with DEAB to inhibit RA. We see reduced cell number

426 in the DEAB treated artery versus the DMSO treated artery (Figure 6E). This

427 corresponds to an increase number of cells in the veins during DEAB treatment

428 compared to DMSO. While we cannot definitively say the migration defects caused the

429 arterial reduction, it indicates that RA is required for the proper formation of the definitive

430 vasculature.
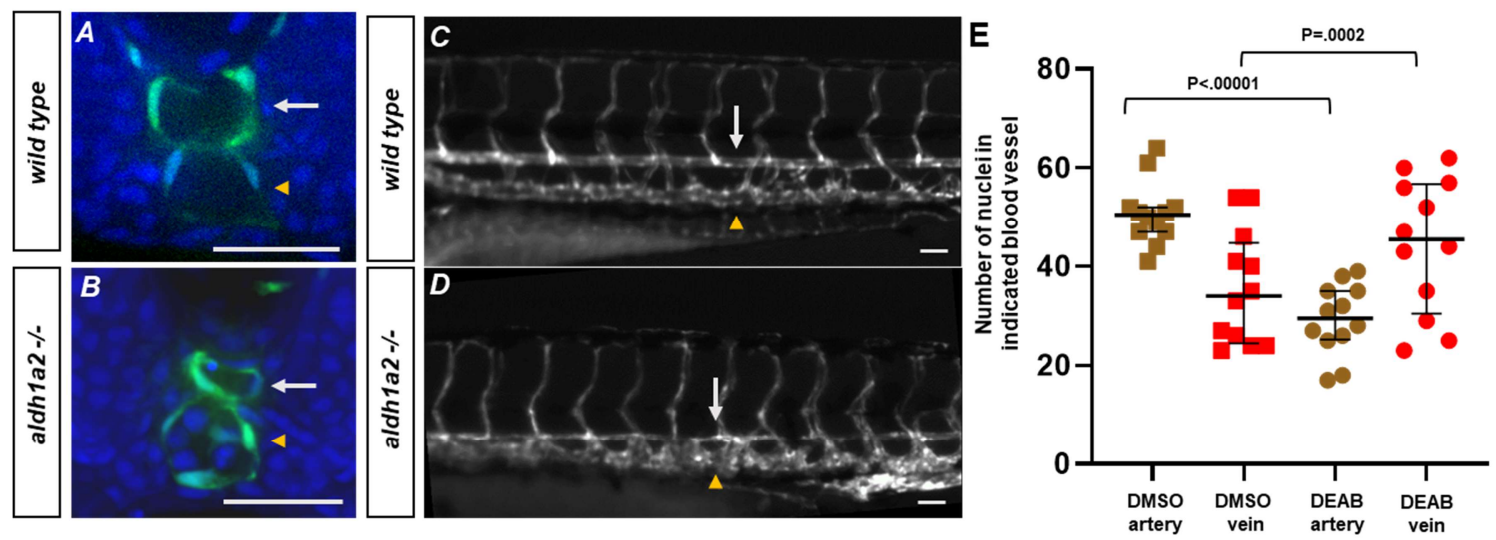

Figure 6. Retinoic acid loss contributes to changes in the definitive vasculature, resulting in large veins and small arteries.

(A) A $5 \mathrm{dpf} t g(k d r l: e G F P)$ embryo. White arrows indicate the dorsal aorta and yellow arrowheads indicate the cardinal vein. (B) $5 \mathrm{dpf} t g(k d r l: e G F P)$, aldh1a2-/- embryo.

White arrows indicate reduced size of dorsal aorta. (C-D) $25 \mathrm{hpf} t g(k d r l: e G F P)$. embryo labeled with DAPI . (C) Wild-type embryos show lumenized blood vessels and normal sized dorsal aorta. (D) Labeled aldh1a2 -/-, tg(kdrl:eGFP) sibling shows small lumenized dorsal aorta with large posterior cardinal vein. (E) Quantification of DMSO $(n=12)$ or $20 \mu$ M DEAB $(n=12)$ treated $t g(k d r l: n / s-e G F P)$ embryos. Nuclei were counted in each structure over a $225 \mu \mathrm{M}$ long transverse section of the zebrafish trunk. Two-tailed $P$-values for the unpaired t-test of wild-type vs $20 \mu \mathrm{M}$ DEAB treated embryos are $<.00001$ and .0002 for arteries and veins, respectively. Scale bars, 50 $\mu \mathrm{m}$. 
432 Retinoic acid induced somite morphogenesis facilitates notochord-endoderm

433 separation and facilitates midline angioblast migration.

435 While NES and midline formation were shown to be highly correlated, we previously

436 showed that cell autonomous RA depletion within the somite caused angioblast

437 migration defects. Therefore, we sought to determine if RA depletion within the somites

438 caused morphological changes that compromised NES and angioblast migration to the

439 midline. During normal development, newly-born somites initially lay flat and extend

440 farther in the medial-lateral axis, demonstrated by a section of 15-somite stage embryo

441 at the $12^{\text {th }}$ somite (Figure 7A, white dashed lines) (Tlili et al., 2019). However, as somites

442 mature, they will narrow in the medial-lateral axis and extend in the dorsal-ventral axis,

443 as demonstrated by a section of the $5^{\text {th }}$ somite of a 15-somite stage embryo (Figure 7B,

444 white dashed line) (Tlili et al., 2019). We therefore sought to determine if somite

445 maturation could be affected cell autonomously in RA depletion conditions. We therefore

446 transplanted either wild-type or HS:dnRAR cells into $\operatorname{tg}(u b b: I c k-m n g$ ) embryos. Embryos

447 were then sectioned at roughly the $5^{\text {th }}$ somite region for 12-somite stage embryos

448 (Figure 7C and 7D). Embryos that were transplanted with wild-type cells showed little

449 difference with the contralateral somite in terms of shape and maturation (Figure 7C,

450 white dashed lines). However, embryos transplanted with HS:dnRAR cells showed a

451 contralateral defect in the somite containing donor cells, with the somite extending

452 farther in the medial-lateral axis while the contralateral somite extends farther in the

453 dorsal-ventral axis (Figure 7D, white dashed lines). To illustrate this effect across

454 multiple experimental embryos, we show overlapping shapes of chimeric wild-type

455 somites, the chimeric HS:dnRAR somites, and the contralateral somites from $H S$ :dnRAR

456 chimeras (Figure 7E). The overlapping images show a consistent trend of a shallow

457 dorsal-ventral axis in the chimeric HS:dnRAR somites compared to the wild-type

458 somites. Together, this indicates that RA is required cell autonomously for somite

459 maturation and dorsal-ventral extension.

461 We speculate that the failure of somites to mature was the result of reduced cell

462 movements within the somite. To confirm this, mosaically labeled cells were generated

463 by transplanting rhodamine dextran labeled cells from a wild-type donor into a wild-type

464 host. The embryos were treated with either DMSO or DEAB and time-lapse imaged over 
465 the course of 4 hours (Figure 7F and Figure 7G, respectively). Cells in DMSO treated

466 embryos showed broad movements consistent with that of somite rotation, where cells

467 located in the anterior of the somite will translocate to the posterior of the somite

468 (Hollway et al., 2007) (Figure 7F, white arrows). However, cells in DEAB treated

469 embryos show little intratissue displacement and cells largely remained static (Figure

$4707 \mathrm{G}$, white arrows). To quantify this, we utilized $\operatorname{tg}($ hsp70l:CAAX-mCherry-2A-NLS-

471 KikGR) embryos to observe cell movement across the entirety of the somite. Embryos

472 were heat shocked during shield stage and subsequently treated with either DMSO or

473 DEAB and their somites were time-lapse imaged for 4 hours. Using Fiji Trackmate

474 software (Tinevez et al., 2017), we quantified the displacement and speed of cell

475 movements within the 2nd somite (Figure $7 \mathrm{H}$ and $7 \mathrm{I}$ ). Cells in DMSO treated embryos

476 showed significant amounts of displacement throughout the somite (Figure $7 \mathrm{H}$ ).

477 However the DEAB cells showed less displacement over time (Figure 7H). Quantification

478 of speed in the same manner showed no statistically significant difference between

479 DMSO and DEAB treatment (Figure 7I). A sample of somite cell tracks shows little

480 directional movement in loss of RA conditions compared to DMSO, indicating cells are

481 not moving in a manner consistent with reshaping of the somite, as well as suggesting a

482 role for RA signaling in somite rotation (Figure 7J and 7K, Supplemental Movie 6 and 7,

483 respectively). The Cxcr4a/Cxcl12a signaling axis has been shown to be responsible for

484 controlling cell movements in the somite. In-situ hybridization for cxcr4a shows a loss of

485 expression in RA depletion conditions and gain of expression in RA addition conditions

486 (Hollway et al., 2007) (Figure S5D and S5E).

488 We speculated that the ventral expansion of the somite that occurs during somite cell

489 rearrangement and maturation was responsible for NES. At the 10 somite stage,

490 embryos have their notochord adjacent to the endoderm and level to ventral-most edge

491 of the somite (Figure $7 \mathrm{~L}$ ). As the embryo develops, the somite expands ventrally (black

492 bracket). As the ventral distance of the somite extends relative to the dorsal part of the

493 notochord (yellow bracket), it induces space that the angioblasts occupy (blue brackets).

494 Starting from the 10 somite stage, we measured the distance of ventral expansion

495 relative to the distance between the notochord and the endoderm every hour for 4 hours.

496 A simple linear regression of the data, indicated by the black line, shows a slope of

4970.002 and $y$-intercept of 1.04 (Figure 7M). The flat slope indicates little variance across

498 time, and the $y$-intercept near 1 indicates that NES is equivalent to ventral expansion of 
499 the somite. The 1:1 correlation of ventral somite expansion and notochord displacement,

500 as well as the $t b \times 16$ loss of function NES defect, provides strong evidence that somite

501 morphogenesis is critical for NES.

502

503 Finally, we investigated a methodology to track all cellular movements within an embryo

504 to co-visualize somite morphogenesis along with angioblast migration and notochord-

505 endoderm separation. We used a live explant method to monitor migration in a

506 transverse section view. Live $t g(k d r l: G F P)$ embryos, injected with mcherry-caax mRNA,

507 were sectioned at the 10 -somite stage at the $4^{\text {th }}$ somite and mounted to observe a

508 transverse section of the trunk. We were able to observe migrating angioblasts, as well

509 as somite morphogenesis and midline NES in the explant (Figure 70-7P, Supplemental

510 Video 8). At the 180' time-point, angioblasts were not at the midline and the hypochord

511 remained flush with the endoderm (Figure 70, Supplemental Video 8). At this time-point

512 the somites have begun to narrow in the medial-lateral axis. By the 240' stage, the

513 hypochord is separated away from the endoderm (Figure 7P). and shortly after this, the

514 angioblasts are able to complete their migration to the midline by the 270 ' time-point

515 (Figure 7P). Similar to the results seen in fixed sections, live explants of embryos treated

516 with DEAB show angioblast migration defects and lack of NES (Supplemental Video 9). 
bioRxiv preprint doi: https://doi.org/10.1101/2021.04.07.438831; this version posted October 21, 2021. The copyright holder for this preprint

(which was not certified by peer review) is the author/funder, who has granted bioRxiv a license to display the preprint in perpetuity. It is made

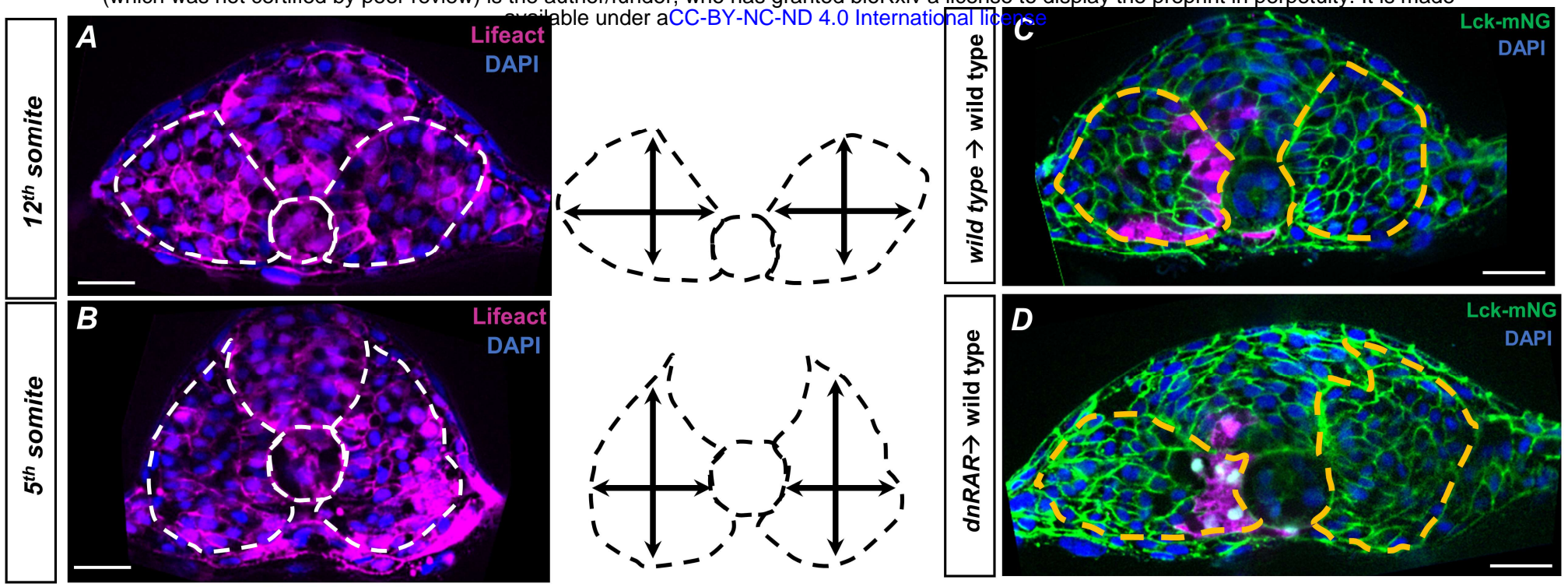

E

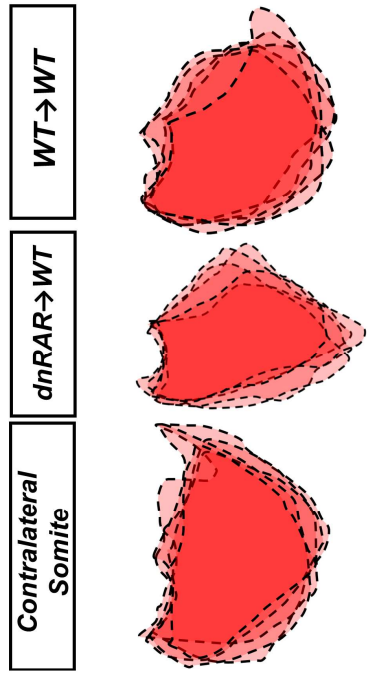

1

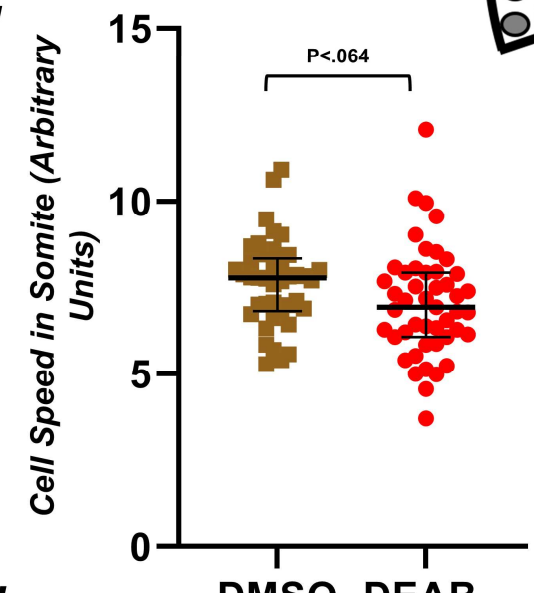

$N$

(5)

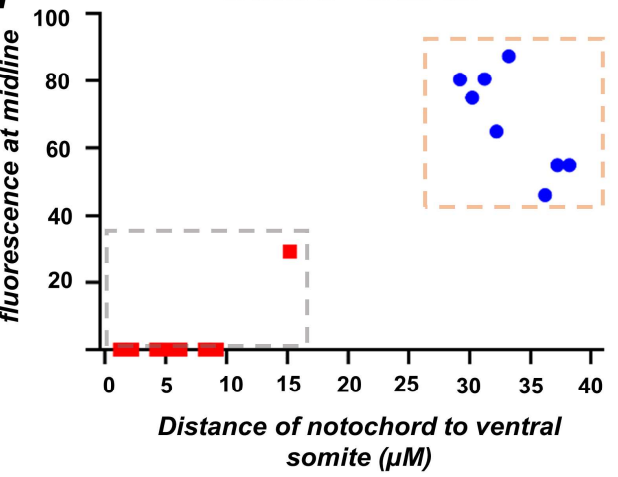

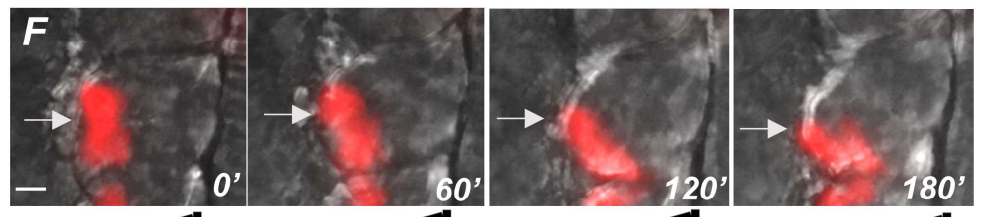
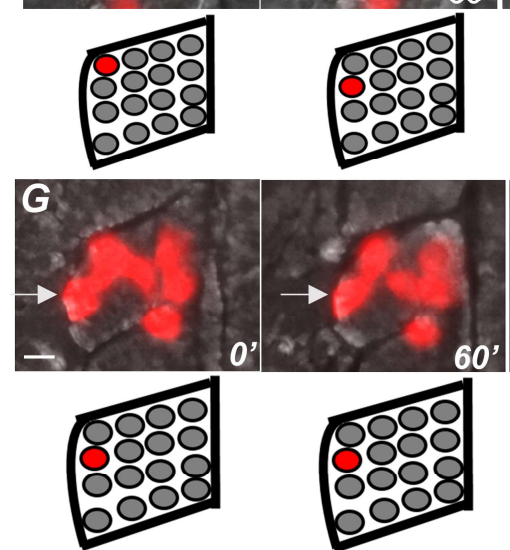

$60^{\circ}$
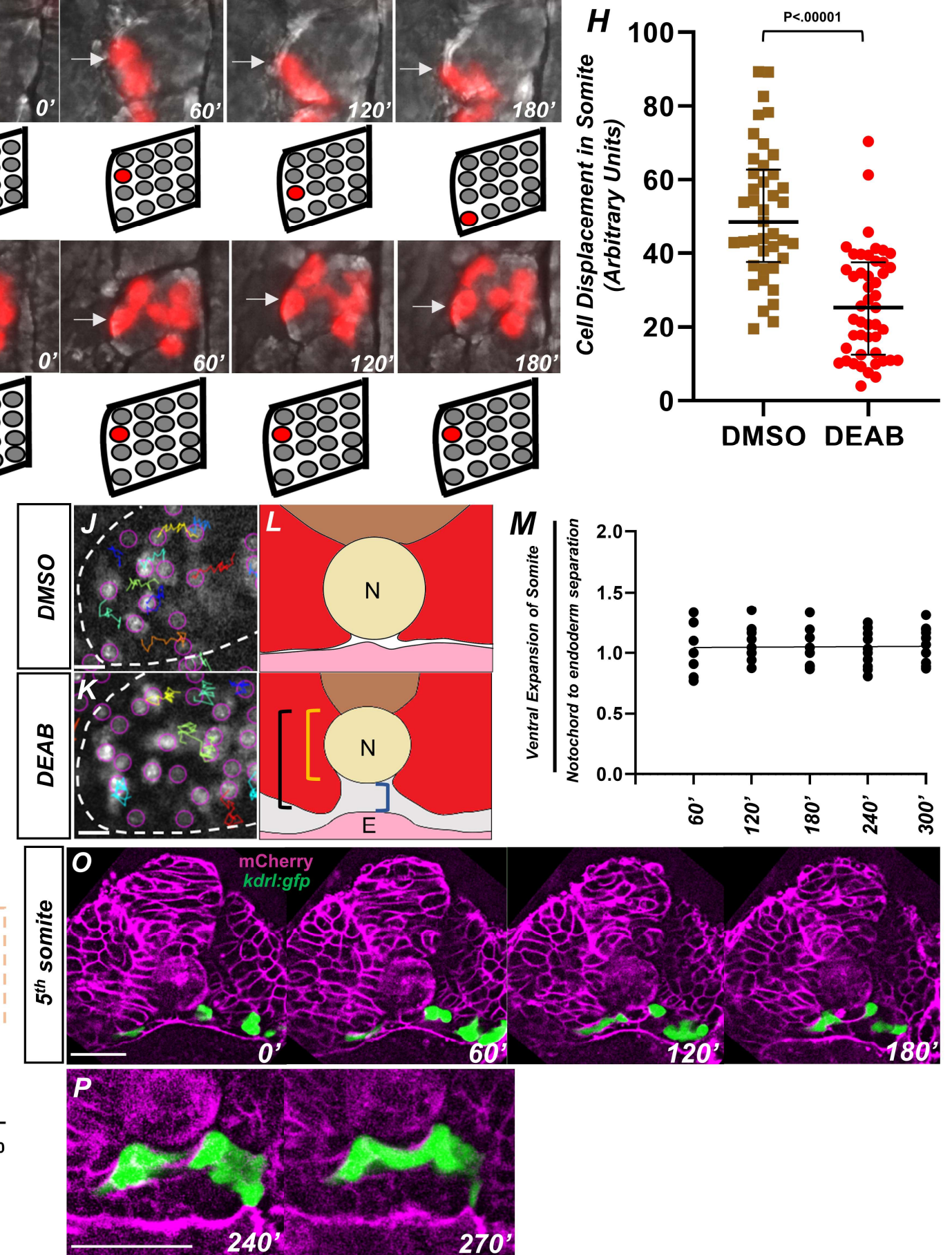


\section{Figure 7. Retinoic acid controls intrasomitic cellular movements and somite shape changes that contribute to angioblast convergence to the midline.}

(A) Image and schematic of newly born $12^{\text {th }}$ somite using $\operatorname{tg}$ (HS:lifeact), $t g(k d r l: e g f p)$ embryo as reference. Dashed lines indicate the somite and notochord. (B) Schematic of more mature $5^{\text {th }}$ somite (at the 12-somite stage) of $t g$ (HS:lifeact), $t g(k d r l: e g f p)$ embryo as reference. Dashed lines indicate somite and notochord. (C) Transplant of wild-type cells into tg(ubb:lck-mng). Dashed lines indicate somite shape. (D) Transplant of $t g$ (HS:dnrar) cells into $\operatorname{tg}(u b b: / c k-m n g)$. Dashed lines indicate somite shape. Note the immature shape of the somite containing transplanted cells. (E) Overlapping somite shapes of either WT $\rightarrow$ WT, $\operatorname{tg}(H S: d n r a r) \rightarrow W T$, or the contralateral somite $\operatorname{tg}($ HS:dnrar) $\rightarrow$ WT. (F-G) Time-lapse images of transplanted rhodaminedextran labeled cells in DMSO and DEAB embryos at 12 somite stage. (F) DMSO treated embryos over 180'. White arrows indicate labeled cells (G) 20

$\mu \mathrm{M}$ DEAB treated embryos over 180'. Scale bars, $50 \mu \mathrm{m}$. Retinoic acid depleted embryos show little cellular movement during somite development.

(H) Quantification of nuclei displacement in the $2^{\text {nd }}$ somite of DMSO and DEAB embryos over a 4 hour time period. Displacement is defined as the distance between the beginning location and end location of the nuclei within the somite 2. (I) Quantification of speed in somite 2, defined as average units over 4 hours in 10 minute increments. (J) Sample of tracks from DMSO treated $t g$ (hsp70l:CAAX-mCherry-2A-NLS-KikGR) embryos. (K) Sample tracks of DEAB treated $t g$ (hsp70I:CAAX-mCherry-2A-NLS-KikGR) embryos. Tracks sort from red to blue, with red being the highest displacement. Scale

bars, $20 \mu \mathrm{m}$ (L) Diagram of ventral somite expansion and notochord to endoderm separation. Ventral somite expansion is calculated by ventral somite length (black bracket) - the notochord diameter (Yellow Bracket).

Notochord/endoderm separation is shown in the blue bracket. (M)

Quantification of the ventral expansion of the somite over notochord/endoderm separation in wild-type embryos. The linear regression

indicated by the black line, where slope $=0.002$, shows ventral somite expansion correlates with notochord/endoderm separation. (N) A two variable graph showing percentage of angioblast fluorescence at the midline compared

to distance of ventral somite expansion beneath the notochord. Blue dots indicate DEAB treated, 15-somite stage embryos sectioned at the 5th somite and red squares are the same embryos sectioned at the 12th somite $(\mathrm{N}=8)$.

Scale bars, $50 \mu \mathrm{m}$. (O) Live section of mcherry-caax injected tg(kdrl:egfp) embryos over 120'. Yellow arrowheads indicate the midline while white arrows indicate angioblasts. (P) Same live section as in (N), except at 240' focused at

the midline. Note the separation of the hypochord and endoderm prior to midline fusion of angioblasts. Scale bars, $50 \mu \mathrm{m}$. 


\section{DISCUSSION}

522 Here, we established a cell nonautonomous angioblast migration model wherein shape

523 changes in the somitic mesoderm create a physical space for angioblasts to migrate into

524 and develop into the axial vasculature. The emergence of the transient VMC is a

525 necessary requirement for contralateral angioblasts to complete their migration and

526 coalesce at the midline. We observed a defect in somitic mesoderm development in RA

527 depletion conditions, which delayed somite maturation, intrasomite cellular movement,

528 and significantly impeded NES. Conversely, we found that addition of RA accelerated

529 somite maturation and NES, along with angioblast migration. We were able to confirm

530 that the somitic mesoderm was required for angioblast migration by examining $t b \times 16$

531 mutants, which have a lack of somites caused by EMT defects (Goto et al., 2017; Ho

532 and Kane, 1990; Manning and Kimelman, 2015; Row et al., 2011). Loss of function of

$533 t b \times 16$ prevented angioblast migration completely and resulted in bifurcated blood vessel

534 formation. These changes in vascular morphology indicate that the somitic mesoderm is

535 a requirement for angioblast migration to the midline. It is notable that in amniotes, such

536 as the mouse and quail, the dorsal aorta and cardinal vein are initially bifurcated in a

537 similar manner to zebrafish tbx16 mutants prior to fusion at the midline (Drake and

538 Fleming, 2000; Pardanaud et al., 1996, 1987). It is possible that evolutionary differences

539 in the somitic mesoderm between teleosts and amniotes could play a role in the

540 differences in vascular development.

542 Very little is known about the process of NES. Our observations indicate that its initiation

543 correlates very closely with angioblast migration, with angioblasts migrating into the

544 VMC seemingly as soon as it appears in both wild-type and RA depletion conditions.

545 Given that these blood vessels become lumenized, and need to achieve a sufficient

546 volume for blood transport, it is plausible that they require morphological changes within

547 the ventral part of the embryo to accommodate for their size. Delays in somite

548 maturation prevent angioblasts from reaching the midline in a normal developmental

549 time frame. It is possible that the consequences of this migration defect results in the

550 malformed blood vessel structure in RA depleted embryos, which have small arteries

551 and larger veins. Prior work showed that the most lateral angioblasts, which migrate later

552 than more medial angioblasts, preferentially adopt a venous fate over an arterial one

553 (Kohli et al., 2013). This implies that somite maturation is a key factor in determining the 
554 proper distribution of endothelial progenitors to the arteries and veins, by essentially

555 timing angioblast migration and altering cell fate decision patterns. However, we cannot

556 definitively determine if this is solely from angioblast migration defects.

558 We found that the formation of the VMC does not necessarily require the notochord

559 tissue itself. In noto morphants, which causes the notochord to adopt a somitic fate,

560 there is still a ventral cavity and angioblasts are able to arrive at the midline. This implies

561 that the notochord is ultimately dispensable for the completion of angioblast migration,

562 even though its loss can produce migration defects (Helker et al., 2015; Sumoy et al.,

563 1997). The migratory defects could be the result of reduced notochord-secreted factors

564 such as apela, which greatly reduce but do not completely abolish migration (Helker et

565 al., 2015). Another factor could be that signals from the notochord, such as Hedgehog,

566 influence somite development which in turn facilitates angioblast migration (Blagden et

567 al., 1997; Hinits et al., 2009; Yin et al., 2018). While the fate of the midline tissue does

568 not need to be notochord for NES to occur, it is likely that mechanical coupling between

569 the somites and midline tissue is required such that somite morphogenesis drives the

570 separation of the midline mesoderm away from the underlying endoderm. In noto loss of

571 function, the somitic tissue that forms at the midline in place of notochord is continuous

572 with the flanking somites. In wild-type embryos, there is evidence of mechanical

573 interactions of the somite and notochord during convergent extension, which are

574 required for the remodeling of the adaxial cells, the medial most cells in the somite (Yin

575 and Solnica-Krezel, 2007).

576

577 The phenotypes observed in both the somites and endothelium after loss of RA signaling 578 are not the result of a simple deficit of mesoderm. RA signaling loss of function affects

579 somite maturation and morphogenesis, but does not prevent them from forming, and yet

580 the migration of the angioblasts is still disrupted. The direct transcriptional targets of RA

581 signaling that induce somite maturation are unknown, but it is clear that RA signaling

582 controls the timing and progress of somite shape changes as they mature. Cell

583 autonomous effects of RA depletion on the somites, coupled with angioblast migration

584 defects in RA depleted somites, indicate RA directly controls somite shape changes that

585 facilitate migration. The fact that somites can adopt mature shapes in explants, even in

586 absence of adjacent somites, indicates this control is local to individual somites. It is

587 also noteworthy that loss of retinoic acid signaling activity in a relatively few number of 
588 adaxially localized cells in our chimeric somites were sufficient to cause somite shape

589 and angioblast migration defects. This implies that the somite cells most medial to the

590 midline could be the population required for the ventral expansion of the somite and

591 NES. It is not clear if NES is required for the initiation of angioblast migration, as some

592 midline-directed cellular movement occurs in DEAB treated embryos before NES is

593 visibly seen. It is possible that the somite itself could facilitate the angioblasts movement

594 to the midline, or simply provide an opening through which angioblasts can reach the

595 midline. The fact that angioblasts reside adjacent to the notochord in $t b \times 16$ mutants, but

596 do not migrate beneath them, implies angioblasts are not sufficient to drive NES or their

597 coalescence at the midline. It is likely that ventral expansions of the somite drives the

598 notochord away from the endoderm. Together, this indicates that somitic maturation

599 requires tight spatial and temporal control to facilitate the concomitant development of

600 the endothelium.

601

\section{Materials and Methods}

603

\section{Generation of the $\operatorname{tg}$ (hsp70l:lifeact-mscarlet) transgenic line}

605 This transgenic line was generated using the plasmid pNJP002 ( $h$ sp70l:lifeact-mScarlet)

606 and to/2 transgenesis (Kawakami, 2004; Kikuta and Kawakami, 2009). pNJP002 was

607 generated using PCR amplification paired with Gibson assembly cloning. A restriction

608 digest was performed on a tol2-hsp70 plasmid (Row et al., 2016) using the restriction

609 enzymes BamHI-HF and Clal (NEB bio labs). PCR amplification of plasmid hCCR4

610 (lifeact-mScarlet) was performed using forward primer

611 CAAGCTACTTGTTCTTTTTGCAGGATCCATGGGCGTGGCCGACTTG and reverse 612 primer TTCGTGGCTCCAGAGAATCGATTCACTTGTACAGCTCGTCCATGC. Gibson

613 Assembly (NEBbuilder HiFi DNA Assembly, NEB bio labs) was performed on restriction

614 digested hCCR2 and PCR amplified lifeact-mscarlet to generate plasmid pNJP002. The

$615 \operatorname{tg}$ (hsp70l:lifeact-mscarlet) line was generated by injecting 25 picograms of pNJP002 with

61625 picograms of tol2 mRNA using methods described previously (Row et al., 2016).

\section{In-situ hybridization and immunohistochemistry}

620 Whole-mount in situ hybridization was performed as previously described (Griffin et al.,

621 1995). An antisense RNA probe was synthesized as previously described for etv2 
622 (Sumanas et al., 2005). The cldn5b, cxcr4a, apela, aplnra, aplnrb, cxcl12a and dab2 DIG

623 probes were generated by taq polymerase-generated PCR fragments integrated into a

624 pCRII vector using the Topo-TA Cloning kit (Thermo Fisher). Immunohistochemistry was

625 performed by fixing embryos overnight in 4\% Paraformaldehyde (Sigma) dissolved in

626 phosphate buffered saline containing $0.1 \%$ Tween-20 (PBST) at $4^{\circ} \mathrm{C}$. Permeabilization

627 was performed by the placing the embryos in PBS containing 2\% Triton X-100 (Sigma)

628 for 1 hour with agitation. Embryos were washed 3x with PBST and blocked in a blocking

629 solution (PBST, 10\% goat serum, 1\% BSA) for 2 hours at room temperature. Primary

630 antibodies, Etv2 Rabbit Polyclonal and GFP mouse monoclonal, were diluted to 1:500 in

631 blocking solution and incubated overnight at $4^{\circ} \mathrm{C}$. Embryos were washed $5 \mathrm{x}$ with PBST

632 for 20 mins each wash. Embryos were then incubated in blocking solution for 2 hours;

633 and then incubated in the secondary antibodies Alexa Fluor 488 and Alexa Fluor 568

634 diluted $1: 1000$ in blocking solution at $4^{\circ} \mathrm{C}$ overnight. DAPI stains were done in a 10

$635 \mu \mathrm{g} / \mathrm{mL}$ dilution in PBST for at least 1 hour with agitation.

636

\section{Histological analysis}

638 Sections were fixed overnight in 4\% Paraformaldehyde (Sigma) dissolved in phosphate

639 buffered saline containing (PBS) at $4^{\circ} \mathrm{C}$. Nuclei labeling was done for whole embryos in

640 a $10 \mu \mathrm{g} / \mathrm{mL}$ dilution of DAPI in PBST for at least 1 hour with agitation. Sections, after

641 fixation and DAPI staining, were done in PBST with a $0.15 \mathrm{~mm}$ microknife (Fine Science

642 Tools), at the indicated somite location for embryos up to $24 \mathrm{hpf}$. At $24 \mathrm{hpf}$ or older,

643 embryos were sectioned at approximately the 7 th somite. Sections were mounted in $1 \%$

644 Agarose in PBS in a in a 35mm glass bottom dish with uncoated \#1.5 coverslip and

$64520 \mathrm{~mm}$ glass diameter (MatTek).

646

647 Microinjections

648 A mix of two tbx16 morpholinos (MO1: AGCCTGCATTATTTAGCCTTCTCTA (1.5 ng)

649 and MO2: GATGTCCTCTAAAAGAAAATGTCAG $(0.75 \mathrm{ng}))$ were prepared as previously

650 reported and injected into $t g(k d r l: G F P)$ embryos (Row et al., 2016). 2.5 ng of control

651 morpholino and noto morpholino were injected into $t g(k d r l: G F P$ ) embryos (Ouyang et al.,

652 2009). $200 \mathrm{ng}$ of mcherry-caax mRNA was injected into $t g(k d r l: G F P)$ embryos.

\section{Microscopy and Imaging}


655 DIC and fluorescent time-lapse images of wild-type, noto morphants, aldh1a2 mutants,

656 tbx16 mutants, and drug treated embryos were performed using a Leica DMI6000B

657 inverted microscope. Wild-type and control embryos were siblings of the morphants and

658 mutants. Live embryos were mounted in 1\% low-melt agarose in embryo media

659 containing $1 \mathrm{x}$ tricaine (25x stock $0.4 \mathrm{~g} /$; Pentair,TRS1) in a $35 \mathrm{~mm}$ glass bottom dish with

660 uncoated \#1.5 coverslip and 20mm glass diameter (MatTek). In-situ hybridization

661 experiments were imaged using a M165FC microscope (Leica) equipped with an Infinity

6623 camera (Lumenera). Embryos were mounted in either horizontal or flat mounted

663 configuration in $70 \%$ glycerol on glass slides.

664

665 Fluorescent images of control, tbx16, and noto morphant (Figure 3 ) cross sections were

666 imaged on a custom assembled spinning disk confocal microscope consisting of an

667 automated Zeiss frame, a Yokogawa CSU-10 spinning disc, a Ludl stage controlled by a

668 Ludl MAC6000 and an ASI filter turret mated to a Photometrics Prime 95B camera. All

669 other cross sections, including explants, were performed on a custom-assembled

670 spinning disk confocal microscope consisting of a Zeiss Imager A.2 frame, a Borealis

671 modified Yokogawa CSU-10 spinning disc, ASI 150uM piezo stage controlled by an

672 MS2000, and ASI filter wheel, and a Hamamatsu ImageEM x2 EMCCD camera

673 (Hamamatsu C9100-23B). These microscopes were controlled with Metamorph

674 microscope control software (V7.10.2.240 Molecular Devices), and laser power levels

675 were set in Vortran's Stradus VersaLase 8 software. Images were processed in Fiji.

677 Drug treatments

678 RA was depleted in embryos using N,N-diethylaminobenzaldehyde (DEAB) and

679 BMS453. Stock concentrations of $20 \mathrm{mM}$ DEAB in DMSO were diluted to working

680 concentrations of $20 \mu \mathrm{M}$ in embryo media. Stock concentrations of $2 \mathrm{mM}$ BMS453 in

681 DMSO were diluted to working concentrations of $2 \mu \mathrm{M}$ in embryo media. Treatments

682 were done on shield stage embryos unless otherwise noted. All-trans RA was used to

683 activate the RA pathway. Stock concentrations of All-trans RA were diluted from $1 \mathrm{mM}$

684 stock to $0.1 \mu \mathrm{M}$ working concentrations in embryo media. All treatments for all-trans RA

685 were performed in tailbud stage.

686

687 Generation of explants for imaging 
688 Transgenic or mutant embryos were grown to the 10 somite stage and then transferred

689 to Modified Barth's Saline (MBS) (Sigma). In this medium, embryos were anesthetized

690 with 1 x Tricaine (25x stock 0.4g/l; Pentair, TRS1) and had their chorions manually

691 removed by forceps. The embryos were then sectioned with a $0.15 \mathrm{~mm}$ microknife (Fine

692 Science Tools). Excess yolk was removed with the microknife, leaving some yolk to

693 prevent injury to the endoderm, and the embryo was then transferred to $35 \mathrm{~mm}$ glass-

694 bottom dish (Matek) with a fire polished pipette. $1.3 \%$ low gelling temperature agarose

695 in MBS was heated to $40^{\circ} \mathrm{C}$ and placed over the embryo in the proper orientation. The

696 agarose was allowed to solidify and the explant was time-lapsed using fluorescent and

697 DIC microscopy.

\section{Quantification and Statistical Analysis}

700 When determining the fluorescence of angioblasts at the midline of the embryo, we

701 utilized the integrated density feature of Fiji software for image analysis. We calculated

702 the corrected total fluorescence of the area at the midline and of the whole embryo,

703 using the notochord as a reference for the midline. Corrected total fluorescence for the

704 midline was calculated as: (mean gray value of midline $x$ area of midline) - (mean gray

705 value of background $x$ area of midline). Corrected total fluorescence for the embryo was

706 calculated: (mean gray value of embryo $x$ area of embryo) - (mean gray value of

707 background $x$ area of embryo). Percentages for fluorescent intensity were calculated as

708 (corrected total fluorescence at the midline) / (corrected total fluorescence of the whole

709 embryo). Tracks for cells were made using the Trackmate plug-in for Fiji (Tinevez et al.,

710 2017). We utilized a Laplacian of Gaussian detector and a Simple LAP Tracker to

711 generate the tracks and analysis. P-values for indicated figures were generated using

712 two-tailed unpaired student t-tests. Graphs were generated using Graphpad Prism 8.4.2

713 for dot plots, and Excel for line graphs. The black lines indicate the median and

714 interquartile ranges, and were generated using the Prism software. Simple linear

715 regression was also determined using Graphpad software.

716

717 Acknowledgments

718

719 We thank David Matus for critical review of the manuscript, as well as members of the

720 Martin and Matus labs for helpful comments. We thank Jesus Torres Vazquez for

721 sending the kdrl transgenic reporter lines. We thank Neal Bhattacharji and Stephanie 
722 Flanagan for excellent zebrafish care. We thank Tianying Chen for assistance in data

723 acquisition. We thank Rebecca Adikes, Thom Geer and Nobska Imaging for microscopy

724 support. The illustration of the 15-somite stage zebrafish embryo was created by

725 Biorender.com. This work was supported by the NSF (IOS 1452928) and NIH NIGMS

726 (R01GM124282).

References

Adikes RC, Kohrman AQ, Martinez MAQ, Palmisano NJ, Smith JJ, Medwig-Kinney TN, Min M, Sallee MD, Ahmed OB, Kim N, Liu S, Morabito RD, Weeks N, Zhao Q, Zhang W, Feldman JL, Barkoulas M, Pani AM, Spencer SL, Martin BL, Matus DQ. 2020. Visualizing the metazoan proliferation-quiescence decision in vivo. Elife 9:174. doi:10.7554/eLife. 63265

Amacher SL, Draper BW, Summers BR, Kimmel CB. 2002. The zebrafish T-box genes no tail and spadetail are required for development of trunk and tail mesoderm and medial floor plate. Development 129:3311-3323.

Balmer S, Nowotschin S, Hadjantonakis AK. 2016. Notochord morphogenesis in mice: Current understanding and open questions. Dev Dyn 245:547-557. doi:10.1002/dvdy.24392

Berenguer M, Lancman JJ, Cunningham TJ, Dong PDS, Duester G. 2018. Mouse but not zebrafish requires retinoic acid for control of neuromesodermal progenitors and body axis extension. Dev Bio/ 441:127-131. doi:10.1016/j.ydbio.2018.06.019 acid signalling: Retinoic acid patterning. Philos Trans $R$ Soc B Biol Sci 375:1809. doi:10.1098/rstb.2019.0556rstb20190556 zebrafish slow muscle mediated by sonic hedgehog. Genes Dev. doi:10.1101/gad.11.17.2163 DYR, Sumanas S. 2017. Vegf signaling promotes vascular endothelial

753 Chng SC, Ho L, Tian J, Reversade B. 2013. ELABELA: A hormone essential for heart 754 development signals via the apelin receptor. Dev Cell 27:672-680.

755 doi:10.1016/j.devcel.2013.11.002 
756 Cleaver O, Krieg P a. 1998. VEGF mediates angioblast migration during development of 757 the dorsal aorta in Xenopus. Development 125:3905-14.

758 Drake CJ, Fleming PA. 2000. Vasculogenesis in the day 6.5 to 9.5 mouse embryo.

$759 \quad$ Blood 95:1671-9. doi:10.1016/j.stem.2010.07.016

760 Duester G. 2008. Retinoic Acid Synthesis and Signaling during Early Organogenesis.

$761 \quad$ Cell. doi:10.1016/j.cell.2008.09.002

762 Emoto Y, Wada H, Okamoto H, Kudo A, Imai Y. 2005. Retinoic acid-metabolizing

763 enzyme Cyp26a1 is essential for determining territories of hindbrain and spinal cord

764 in zebrafish. Dev Biol. doi:10.1016/j.ydbio.2004.11.023

765 Fouquet B, Weinstein BM, Serluca FC, Fishman MC. 1997. Vessel patterning in the embryo of the zebrafish: Guidance by notochord. Dev Biol 183:37-48. doi:10.1006/dbio.1996.8495

Freyer L, Hsu CW, Nowotschin S, Pauli A, Ishida J, Kuba K, Fukamizu A, Schier AF, Hoodless PA, Dickinson ME, Hadjantonakis AK. 2017. Loss of Apela Peptide in Mice Causes Low Penetrance Embryonic Lethality and Defects in Early Mesodermal Derivatives. Cell Rep 20:2116-2130. doi:10.1016/j.celrep.2017.08.014

Glickman NS, Kimmel CB, Jones MA, Adams RJ. 2003. Shaping the zebrafish notochord. Development 130:873-887. doi:10.1242/dev.00314

Goto H, Kimmey SC, Row RH, Matus DQ, Martin BL. 2017. FGF and canonical Wnt signaling cooperate to induce paraxial mesoderm from tailbud neuromesodermal progenitors through regulation of a two-step epithelial to mesenchymal transition. Development 144:1412-1424. doi:10.1242/dev.143578

Griffin KJ, Amacher SL, Kimmel CB, Kimelman D. 1998. Molecular identification of spadetail: regulation of zebrafish trunk and tail mesoderm formation by T-box genes. Development 125:3379-3388.

Halpern ME, Thisse C, Ho RK, Thisse B, Riggleman B, Trevarrow B, Weinberg ES, Postlethwait JH, Kimmel CB. 1995. Cell-autonomous shift from axial to paraxial mesodermal development in zebrafish floating head mutants. Development 121:4257-4265. doi:10.1016/0168-9525(96)81431-4

Hamade A, Deries M, Begemann G, Bally-Cuif L, Genêt C, Sabatier F, Bonnieu A, Cousin X. 2006. Retinoic acid activates myogenesis in vivo through Fgf8 signalling.

788 Heine UI, Roberts AB, Munoz EF, Roche NS, Sporn MB. 1986. Effects of retinoid deficiency on the development of the heart and vascular system of the quail 
embryo. Virchows Arch B Cell Pathol Incl Mol Pathol. doi:10.1007/BF02889897

Helker CSM, Schuermann A, Pollmann C, Chng SC, Kiefer F, Reversade B, Herzog W. 2015. The hormonal peptide Elabela guides angiob lasts to the midline during vasculogenesis. Elife 4:1-13. doi:10.7554/eLife.06726

Hinits Y, Osborn DPS, Hughes SM. 2009. Differential requirements for myogenic regulatory factors distinguish medial and lateral somitic, cranial and fin muscle fibre populations. Development 136:403-414. doi:10.1242/dev.028019

Ho RK, Kane DA. 1990. Cell-autonomous action of zebrafish spt-1 mutation in specific mesodermal precursors. Nature. doi:10.1038/348728a0

Hogan KA, Bautch VL. 2004. Blood Vessel Patterning at the Embryonic Midline. Curr Top Dev Biol 62:55-85.

Hollway GE, Bryson-Richardson RJ, Berger S, Cole NJ, Hall TE, Currie PD. 2007. Whole-Somite Rotation Generates Muscle Progenitor Cell Compartments in the Developing Zebrafish Embryo. Dev Cell 12:201-219. doi:10.1016/j.devcel.2007.01.001

Isogai S, Horiguchi M, Weinstein BM. 2001. The vascular anatomy of the developing zebrafish: An atlas of embryonic and early larval development. Dev Biol 230:278301. doi:10.1006/dbio.2000.9995 $\mathrm{J}$, Blumberg B. 2014. Active repression by RAR signaling is required for vertebrate

811 Janesick A, Tang W, Shioda T, Blumberg B. 2018. RARy is required for mesodermal

812 gene expression prior to gastrulation in xenopus. Dev 145:2-9.

813 doi:10.1242/dev.147769

814 Jin SW, Beis D, Mitchell T, Chen JN, Stainier DYR. 2005. Cellular and molecular

815 analyses of vascular tube and lumen formation in zebrafish. Development

816 132:5199-5209. doi:10.1242/dev.02087

817 Kawakami K. 2004. Transgenesis and Gene Trap Methods in Zebrafish by Using the

818 Tol2 Transposable Element. Methods Cell Biol 77:201-222. doi:10.1016/S0091-

$819 \quad 679 \times(04) 77011-9$

820 Kikuta H, Kawakami K. 2009. Transient and stable transgenesis using tol2 transposon

821 vectors. Methods Mol Biol 546:69-84. doi:10.1007/978-1-60327-977-2_5

822 Kimelman D. 2016. Tales of tails (and trunks): forming the posterior body in vertebrate 823 embryos David Kimelman. Curr Top Dev Biol 116:517-536. 
doi:10.1016/bs.ctdb.2015.12.008.Tales

825 Kimmel CB, Ballard WW, Kimmel SR, Ullmann B, Schilling TF. 1995. Stages of

826 embryonic development of the zebrafish. Dev Dyn 203:253-310.

827 doi:10.1002/aja.1002030302

828 Kimmel CB, Kane DA, Walker C, Warga RM, Rothman MB. 1989. A mutation that changes cell movement and cell fate in the zebrafish embryo. Nature. doi:10.1038/337358a0

Kohli V, Schumacher JA, Desai SP, Rehn K, Sumanas S. 2013. Arterial and Venous Progenitors of the Major Axial Vessels Originate at Distinct Locations. Dev Cell 25:196-206. doi:10.1016/j.devcel.2013.03.017

Kumar S, Duester G. 2014. Retinoic acid controls body axis extension by directly repressing Fgf8 transcription. Dev 141:2972-2977. doi:10.1242/dev.112367

Lawson ND, Vogel AM, Weinstein BM. 2002. Sonic hedgehog and vascular endothelial growth factor act upstream of the Notch pathway during arterial endothelial differentiation. Dev Cell 3:127-136. doi:10.1016/S1534-5807(02)00198-3 2014. The Role of Sdf-1a signaling in Xenopus laevis somite morphogenesis. Dev Dyn. doi:10.1002/dvdy.24092 2015. Zebrafish foxc1a plays a crucial role in early somitogenesis by restricting the expression of aldh1a2 directly. J Biol Chem 290:10216-10228.

846 Lohnes D, Mark M, Mendelsohn C, Dolle P, Dierich A, Gorry P, Gansmuller A, Chambon

847 P. 1994. Function of the retinoic acid receptors (RARs) during development. (I) Craniofacial and skeletal abnormalities in RAR double mutants. Development 120:2723-2748. JS. 2013. Transgenic retinoic acid sensor lines in zebrafish indicate regions of available embryonic retinoic acid. Dev Dyn 242:989-1000. doi:10.1002/dvdy.23987 cell migration of zebrafish mesodermal progenitors. Dev Biol 406:172-185. doi:10.1016/j.ydbio.2015.09.001

Martin BL. 2016. Factors that coordinate mesoderm specification from neuromesodermal progenitors with segmentation during vertebrate axial extension. Semin Cell Dev 
Biol 49:59-67. doi:10.1016/j.semcdb.2015.11.014

Martin BL, Kimelman D. 2012. Canonical Wnt Signaling Dynamically Controls Multiple Stem Cell Fate Decisions during Vertebrate Body Formation. Dev Cell 22:223-232. doi:10.1016/j.devcel.2011.11.001 progenitor niche. Genes Dev 24:2778-2783. doi:10.1101/gad.1962910 McMillen P, Holley SA. 2015. The tissue mechanics of vertebrate body elongation and segmentation. Curr Opin Genet Dev. doi:10.1016/j.gde.2015.02.005

Mendelsohn C, Lohnes D, Decimo D, Lufkin T, LeMeur M, Chambon P, Mark M. 1994. Function of the retinoic acid receptors (RARs) during development. (II) Multiple abnormalities at various stages of organogenesis in RAR double mutants. Development 120:2749-2771. diethylaminobenzaldehyde (DEAB) as a substrate and mechanism-based inhibitor for human ALDH isoenzymes. Chem Biol Interact 234:18-28. doi:10.1016/j.cbi.2014.12.008

Niederreither K, Vermot J, Messaddeq N, Schuhbaur B, Chambon P, Dollé P. 2001. Embryonic retinoic acid synthesis is essential for heart morphogenesis in the mouse. Development 128:1019-1031.

Norris ML, Pauli A, Gagnon JA, Lord ND, Rogers KW, Mosimann C, Zon LI, Schier AF. 2017. Toddler signaling regulates mesodermal cell migration downstream of nodal signaling. Elife 6:e22626. doi:10.7554/eLife.22626

Ouyang X, Shestopalov IA, Sinha S, Zheng G, Pitt CLW, Li WH, Olson AJ, Chen JK. 2009. Versatile synthesis and rational design of caged morpholinos. J Am Chem Soc 131:13255-13269. doi:10.1021/ja809933h

Pardanaud L, Altmann C, Kitos P, Dieterlen-Lievre F, Buck CA. 1987. Vasculogenesis in the early quail blastodisc as studied with a monoclonal antibody recognizing endothelial cells. Development 100:339-349.

Pardanaud L, Luton D, Prigent M, Bourcheix LM, Catala M, Dieterlen-Lièvre F. 1996. Two distinct endothelial lineages in ontogeny, one of them related to hemopoiesis. Development 122:1363-1371.

Pauli A, Norris ML, Valen E, Chew GL, Gagnon JA, Zimmerman S, Mitchell A, Ma J, Dubrulle J, Reyon D, Tsai SQ, Joung JK, Saghatelian A, Schier AF. 2014. Toddler: An embryonic signal that promotes cell movement via apelin receptors. Science 
892

893

894

895

896

897

898

899

900

901

902

903

904

905

906

907

908

909

910

911

912

913

914

915

916

917

918

919

920

921

922

923

924

925

(80- ) 343. doi:10.1126/science. 1248636

Pillay LM, Mackowetzky KJ, Widen SA, Waskiewicz AJ. 2016. Somite-derived retinoic acid regulates zebrafish hematopoietic stem cell formation. PLoS One. doi:10.1371/journal.pone.0166040

Riedl J, Crevenna AH, Kessenbrock K, Yu JH, Neukirchen D, Bista M, Bradke F, Jenne D, Holak TA, Werb Z, Sixt M, Wedlich-Soldner R. 2008. Lifeact: A versatile marker to visualize F-actin. Nat Methods 5:605-607. doi:10.1038/nmeth.1220

Row RH, Maître JL, Martin BL, Stockinger P, Heisenberg CP, Kimelman D. 2011. Completion of the epithelial to mesenchymal transition in zebrafish mesoderm requires Spadetail. Dev Biol 354:102-110. doi:10.1016/j.ydbio.2011.03.025

Row RH, Pegg A, Kinney B, Farr GH, Maves L, Lowell S, Wilson V, Martin BL. 2018. BMP and FGF signaling interact to pattern mesoderm by controlling basic helixloop-helix transcription factor activity. Elife 7:e31018. doi:10.7554/eLife.31018

Row RH, Tsotras SR, Goto H, Martin BL. 2016. The zebrafish tailbud contains two independent populations of midline progenitor cells that maintain long-term germ layer plasticity and differentiate in response to local signaling cues. Dev. doi:10.1242/dev.129015

Sandell LL, Sanderson BW, Moiseyev G, Johnson T, Mushegian A, Young K, Rey JP, Ma JX, Staehling-Hampton K, Trainor PA. 2007. RDH10 is essential for synthesis of embryonic retinoic acid and is required for limb, craniofacial, and organ development. Genes Dev 21:1113-1124. doi:10.1101/gad.1533407

Shaw KM, Castranova DA, Pham VN, Kamei M, Kidd KR, Lo BD, Torres-Vasquez J, Ruby A, Weinstein BM. 2006. Fused-Somites-Like Mutants Exhibit Defects in Trunk Vessel Patterning. Dev Dyn 235:1753-1760. doi:10.1002/dvdy.20814

Skvarca LB, Han HI, Espiritu EB, Missinato MA, Rochon ER, McDaniels MD, Bais AS, Roman BL, Waxman JS, Watkins SC, Davidson AJ, Tsang M, Hukriede NA. 2019. Enhancing regeneration after acute kidney injury by promoting cellular dedifferentiation in zebrafish. DMM Dis Model Mech. doi:10.1242/dmm.037390

Stern CD, Piatkowska AM. 2015. Multiple roles of timing in somite formation. Semin Cell Dev Biol 42:134-139. doi:10.1016/j.semcdb.2015.06.002

Sumanas S, Jorniak T, Lin S. 2005. Identification of novel vascular endothelial-specific genes by the microarray analysis of the zebrafish cloche mutants. Blood 106:534541. doi:10.1182/blood-2004-12-4653

Sumoy L, Bennett Keasey J, Dittman TD, Kimelman D. 1997. A role for notochord in 
926

927

928

929

930

931

932

933

934

935

936

937

938

939

940

941

942

943

944

945

946

947

948

949

950

951

952

953

954

955

956

957

958

959

axial vascular development revealed by analysis of phenotype and the expression of VEGR-2 in zebrafish flh and ntl mutant embryos. Mech Dev 63:15-27. doi:10.1016/S0925-4773(97)00671-0

Talbot WS, Trevarrow B, Halpern ME, Melby AE, Farr G, Postlethwait JH, Jowett T, Kimmel CB, Kimelman D. 1995. A homeobox gene essential for zebrafish notochord development. Nature 378:150-157. doi:10.1038/378150a0

Tani S, Chung U il, Ohba S, Hojo H. 2020. Understanding paraxial mesoderm development and sclerotome specification for skeletal repair. Exp Mol Med 52:1166-1177. doi:10.1038/s12276-020-0482-1

Therapontos C, Vargesson N. 2010. Zebrafish notch signalling pathway mutants exhibit trunk vessel patterning anomalies that are secondary to somite misregulation. Dev Dyn 239:2761-2768. doi:10.1002/dvdy.22410

Thompson MA, Ransom DG, Pratt SJ, MacLennan H, Kieran MW, Detrich HW, Vail B, Huber TL, Paw B, Brownlie AJ, Oates AC, Fritz A, Gates MA, Amores A, Bahary N, Talbot WS, Her H, Beier DR, Postlethwait JH, Zon LI. 1998. The cloche and spadetail genes differentially affect hematopoiesis and vasculogenesis. Dev Biol. doi:10.1006/dbio.1998.8887

Tinevez JY, Perry N, Schindelin J, Hoopes GM, Reynolds GD, Laplantine E, Bednarek SY, Shorte SL, Eliceiri KW. 2017. TrackMate: An open and extensible platform for single-particle tracking. Methods 115:80-90. doi:10.1016/j.ymeth.2016.09.016

Tlili S, Yin J, Rupprecht JF, Mendieta-Serrano MA, Weissbart G, Verma N, Teng X, Toyama Y, Prost J, Saunders TE. 2019. Shaping the zebrafish myotome by intertissue friction and active stress. Proc Natl Acad Sci U S A 116:25430-25439. doi:10.1073/pnas.1900819116

Torres-Vázquez J, Gitler AD, Fraser SD, Berk JD, Pham VN, Fishman MC, Childs S, Epstein JA, Weinstein BM. 2004. Semaphorin-plexin signaling guides patterning of the developing vasculature. Dev Cell 7:117-123. doi:10.1016/j.devcel.2004.06.008

Yabe T, Takada S. 2016. Molecular mechanism for cyclic generation of somites: Lessons from mice and zebrafish. Dev Growth Differ 31-42. doi:10.1111/dgd.12249

Yin C, Solnica-Krezel L. 2007. Convergence and extension movements affect dynamic notochord-somite interactions essential for zebrafish slow muscle morphogenesis. Dev Dyn 236:2742-2756. doi:10.1002/dvdy.21295

Yin J, Lee R, Ono Y, Ingham PW, Saunders TE. 2018. Spatiotemporal Coordination of FGF and Shh Signaling Underlies the Specification of Myoblasts in the Zebrafish 
960 Embryo. Dev Cell 46:735-750.e4. doi:10.1016/j.devcel.2018.08.024

961 Youn BW, Malacinski GM. 1981. Somitogenesis in the amphibian Xenopus laevis:

962 scanning electron microscopic analysis of intrasomitic cellular arrangements during

963 somite rotation. J Embryol Exp Morphol 64:23-43.

964

965

966

967

968

969

970

971

972

973

974

975

976

977

978

979

980

981

982

983

984

985

986

987

988

989

990

991

992

993 


\section{Supplemental Figures}

995
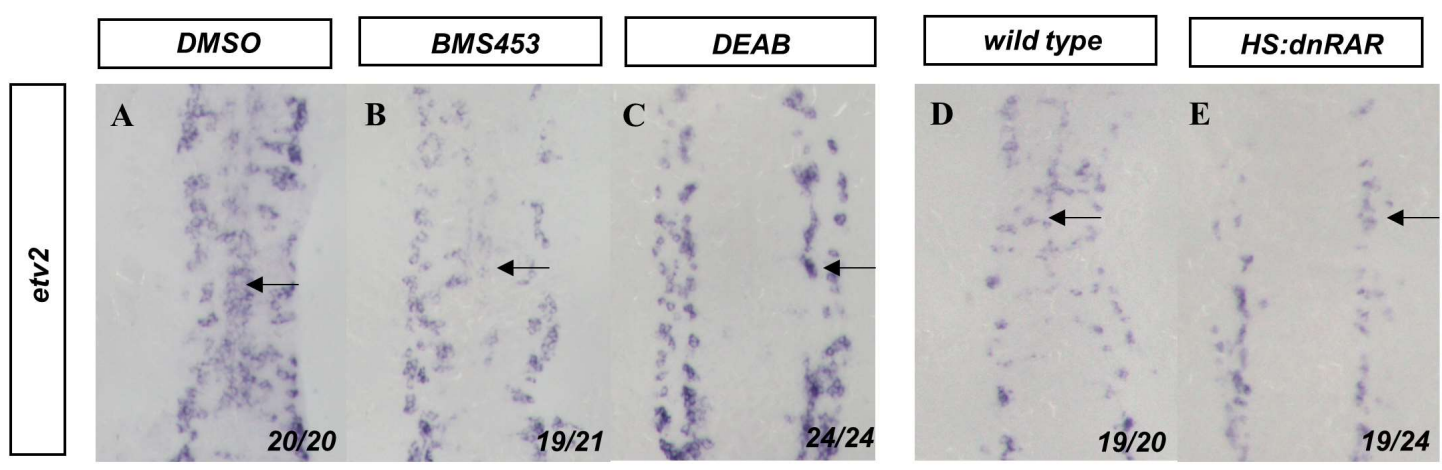

Figure S1. Three alternative methods of RA signaling disruption inhibit midline angioblast migration.

Angioblasts are labelled by etv2 in-situ hybridization in 13-somite stage embryos (black arrows) after (A) DMSO, (B) BMS453, or (C) DEAB treatment, or after bud stage heat-shock in (D) wild-type and (E) HS:dnrar embryos. Embryos are shown from a dorsal view with anterior to the top. 

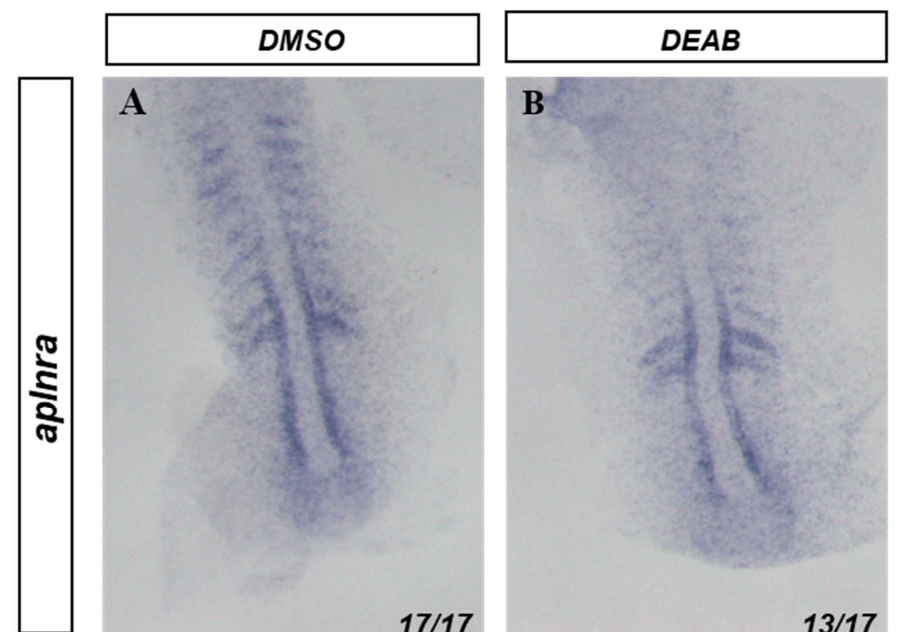

$17 / 17$
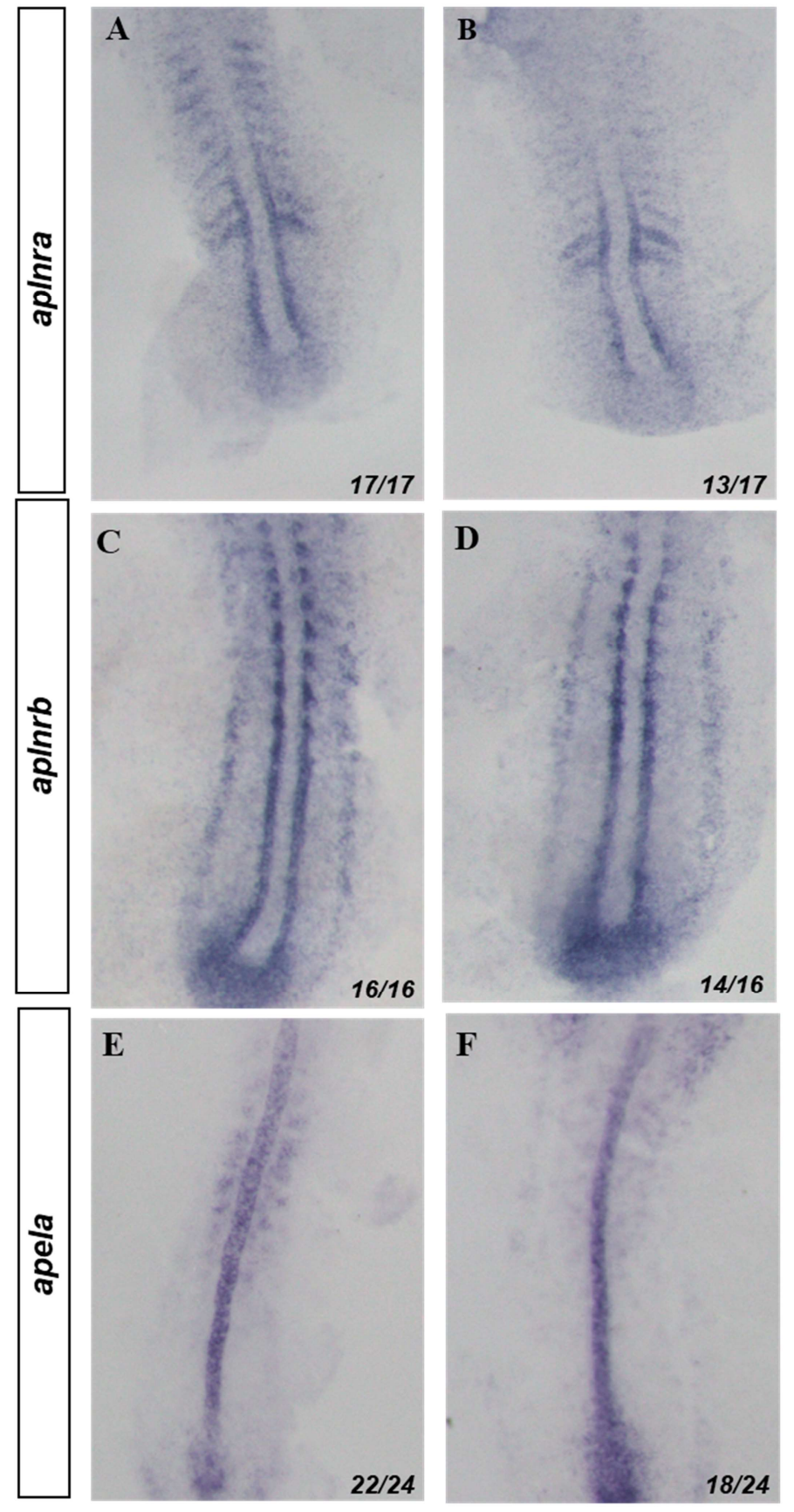

Figure S2. Apela/Aplnr axis is not altered by changes in retinoic acid signaling In situ hybridization of 10-somite stage embryos. (A-B) In-situ hybridization against aplnra. (A) DMSO treated embryos. (B) DEAB treated embryos. (C-D) Insitu hybridization against aplnrb. (C) DMSO treated embryos (D) DEAB treated embryos. (E-F) In-situ hybridization against apela. (E) DMSO treated embryos

(F) DEAB treated embryos 

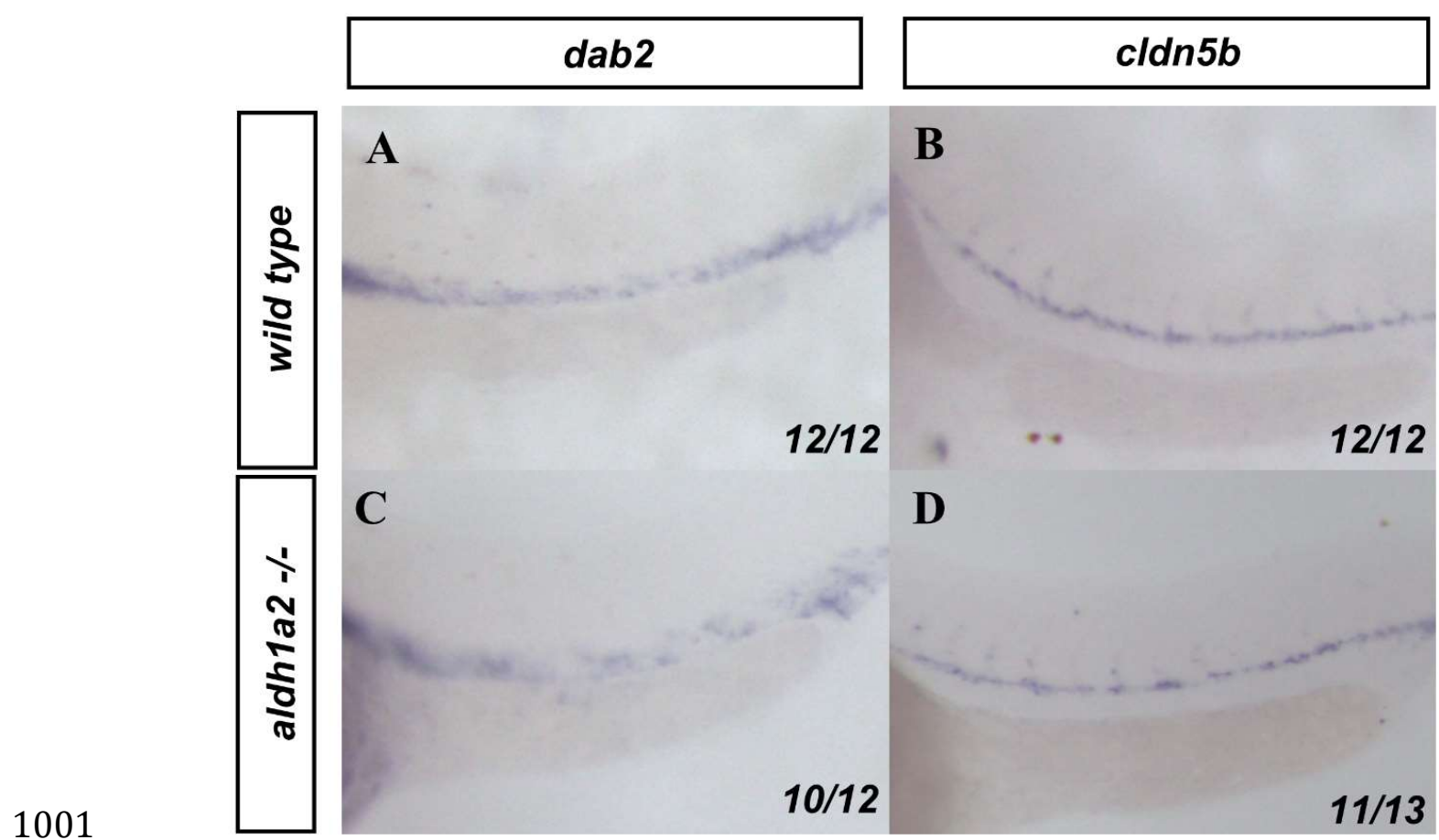

$12 / 12$

B

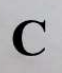

$12 / 12$

\section{D}

Figure S3. Effects of RA depletion on arterial and venous markers.

$(\mathrm{A}, \mathrm{C}) \mathrm{dab} 2$ and $(\mathrm{B}, \mathrm{D}) \mathrm{cldn} 5 \mathrm{~b}$ in-situ hybridization labels the veins and arteries, respectively in (A-B) wild-type or (C-D) aldh1a2 -/- embryos. Embryos are shown from a lateral view with anterior to the left.
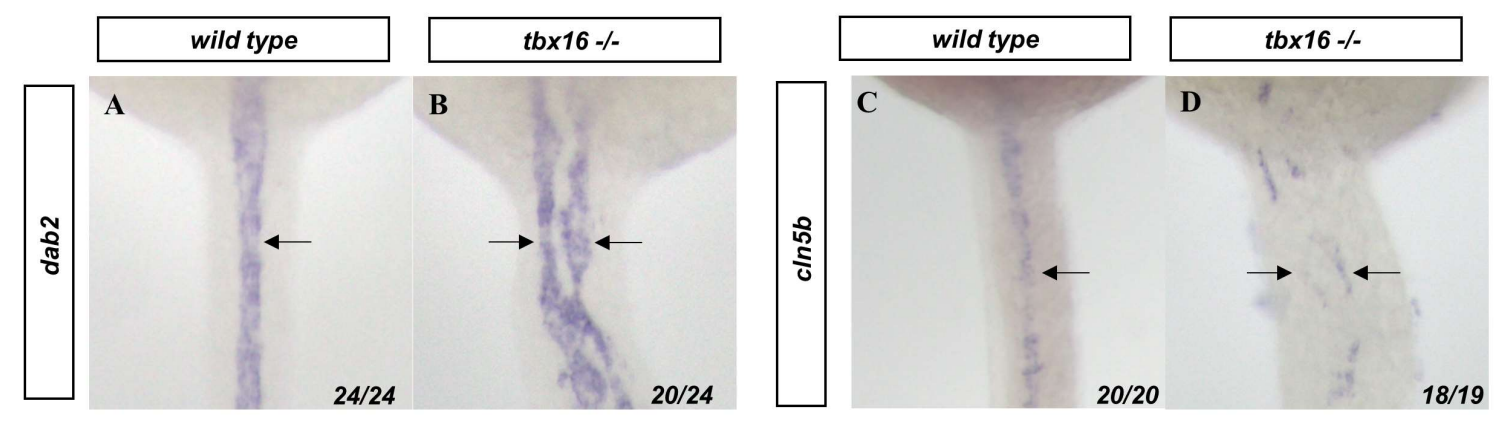

Figure S4. Bifurcated angioblasts in tbx16 mutants preferentially join the venous population. (A, B) dab2 and $(C, D) c l d n 5 b$ in-situ hybridization labels the veins and arteries, respectively (black arrows) in (A,C) wild-type and (B,D) tbx16 -/embryos. Embryos are shown from a dorsal view with anterior to the top. 


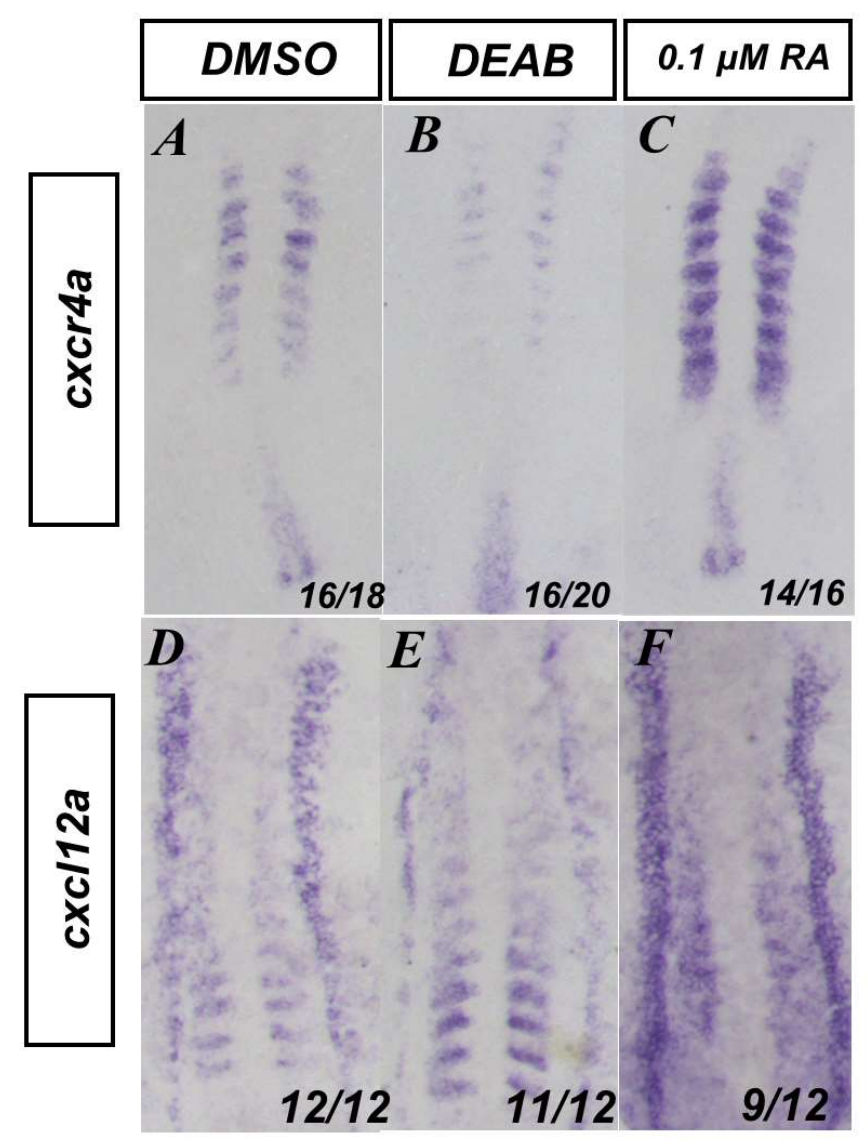

Figure S5. Retinoic acid signaling affects the expression of genes associated with somitic movements.

(A-C) In-situ hybridization for cxcr4a and (D-F) cxcl12a in (A, D) DMSO, (B, E) $20 \mu \mathrm{M}$ $\mathrm{DEAB}$, or $(\mathbf{C}, \mathbf{F}) 0.1 \mu \mathrm{M}$ RA treated embryos. Embryos are shown from a dorsal view with anterior to the top.

1008 Movie S1. Time-lapse fluorescent imaging of $t g(k d r l: e G F P)$ embryo at the 10

1009 somite-stage. $\operatorname{tg}(k d r l: e G F P)$ marks angioblasts as they migrate to the midline.

1010 Angioblasts display the anterior posterior processivity while coalescing at the midline.

1011 Frame rate $=$ one image $/ 5 \mathrm{~min}$. Run time $=235 \mathrm{~min}$.

1013 Movie S2. Time-lapse fluorescent imaging of $t g(k d r l: e G F P)$, aldh1a2 -/- embryo at

1014 the 10 somite-stage. $\operatorname{tg}(\mathrm{kdrl}: \mathrm{e} G F P)$ marks angioblasts as they migrate to the midline.

1015 Angioblasts lose anterior to posterior processivity and show disrupted migration. Frame

1016 rate $=$ one image $/ 5 \mathrm{~min}$. Run time $=235 \mathrm{~min}$. 
Movie S3. Time-lapse fluorescent imaging of a 10 somite-stage tg(kdrl:eGFP) embryo with addition of $0.1 \mu \mathrm{M}$ RA at the tailbud stage. $\operatorname{tg}(k d r l: e G F P)$ marks angioblasts as they migrate to the midline. Angioblasts accelerate their processivity towards the midline. Frame rate $=$ one image $/ 5 \mathrm{~min}$. Run time $=235 \mathrm{~min}$. tg(kdrl:eGFP) host. Cells expressing HS:id3, HS; dnRAR migrate to the midline along with angioblasts in a $t g(k d r l: e G F P)$ host. Cells with dnRAR migrate faithfully with angioblasts. Frame rate= one image $/ 5 \mathrm{~min}$. Run time $=180 \mathrm{~min}$.

Movie S5. Time-lapse fluorescent imaging of tg(tbxta:kaedae), $\operatorname{tg}($ actc1b:gfp) trunk explant. Trunk explant shows notochord displacement away from ventral-most portion of somites. Frame rate= one image $/ 15 \mathrm{~min}$. Run time= $285 \mathrm{~min}$. NLS-kikume embryo in DMSO treatment. HS:mcherry-caax-p2a-NLS-kikume marks nuclei within the somite. Sample tracks ranging from red (most displacement) to blue

1037 (least displacement) show broad movement within the somite. Frame rate= one image/5 $1038 \min$. Run time $=240 \mathrm{~min}$.

1040 Movie S7. Time-lapse confocal fluorescent imaging of a HS:mcherry-caax-p2a-

1041 NLS-kikume embryo in DEAB treatment. HS:mcherry-caax-p2a-NLS-kikume marks

1042 nuclei within the somite. Sample tracks ranging from red (most displacement) to blue

1043 (least displacement) show little directional movement in $20 \mu \mathrm{M}$ DEAB treatment

1044 conditions. Frame rate $=$ one image $/ 5 \mathrm{~min}$. Run time= $240 \mathrm{~min}$.

1046 Movie S8. Time-lapse fluorescent movie of mcherry-caax mRNA injected

1047 tg(kdrl:eGFP) explant at the 10 somite-stage. $\operatorname{tg}(k d r l: e G F P)$ marks angioblasts as

1048 they migrate to the midline and mcherry-caax marks the cell surfaces. A trunk explant, 1049 imaged around the 5th somite, shows normal migration of the angioblasts to the midline.

1050 Frame rate $=$ one image $/ 5 \mathrm{~min}$. Run time $=280 \mathrm{~min}$. 
1052 Movie S9. Time-lapse fluorescent movie of mcherry-caax mRNA injected

$1053 \operatorname{tg}(k d r l: e G F P)$ explant at the 10 somite-stage, treated with $20 \mu M$ DEAB.

$1054 \operatorname{tg}(k d r l: e G F P)$ marks angioblasts as they migrate to the midline. The trunk explant shows

1055 angioblast migration defects seen in fixed DEAB sections. Frame rate $=$ one image $/ 5 \mathrm{~min}$.

1056 Run time $=175 \mathrm{~min}$.

1057 